\title{
STABILITY BEHAVIOR OF THREE NON-NEWTONIAN MAGNETIC FLUIDS IN POROUS MEDIA*
}

\author{
KADRY ZAKARIA ${ }^{\dagger}$, MAGDY A. SIRWAH ${ }^{\ddagger}$, AND SAMEH A. ALKHARASHI ${ }^{\S}$
}

\begin{abstract}
The present study deals with stability properties of two-dimensional non-Newtonian fluid layers moving in porous media, under the influence of uniform magnetic field. The system is composed of a middle fluid embedded between two semi-infinite fluids. The limiting case of the stability of one interface between two viscoelastic fluids is discussed. The presented analysis takes into account the modified Darcy's law. The principle aim of this work is to investigate the influence of fluid elasticity and the porosity effect on the growth rate in the presence of a magnetic field. The stability analysis is performed theoretically and stability diagrams are obtained. The stability analysis shows that non-Newtonian (viscoelastic) fluid layers have a higher growth rate than Newtonian fluid layers, indicating that non-Newtonian fluid sheets are more unstable than Newtonian fluid sheets. It is observed that in fluid sheets the fluid elasticity tends to damp the stability, whereas the fluid viscosity results in an enhancement of stability. The phenomenon of the dual role is found to increase the porous parameter as well as the magnetic permeability ratio. It has been found that the increase of the viscosity coefficient damps the growth rate, while increasing the Reynolds number has the opposite effect.
\end{abstract}

Key words. Modified Darcy's law, non-Newtonian fluids, three layers stability, porous media.

AMS subject classifications. 76 (Fluid mechanics).

\section{Introduction}

Interest in flows of non-Newtonian fluids through a porous medium has grown considerably because of their applications in modern technology and industries, such as in diesel engines, gas turbine engines, liquid rocket engines, oil burners, spray coating processes, plastics manufacturing, and metal powder production and lubrication.

A series of studies on hydrodynamic stability has been initiated by many authors; in particular, Funada and Joseph [1] have discussed the instability of viscous potential flow in a horizontal rectangular channel. The analysis leads to an explicit dispersion relation in which the effects of surface tension and viscosity on the normal stress are not neglected but the effect of shear stresses is. The influence of viscosity on the stability of the plane interface separating two incompressible superposed fluids of uniform densities, when the whole system is immersed in a uniform horizontal magnetic field, has been studied by Bhatia [2]. He has developed the stability analysis for two fluids of equal kinematic viscosities and different uniform densities. A good account of hydrodynamic stability problems has also been given by Drazin and Reid [3] and Joseph [4]. The unsteady electrohydrodynamic stability has been investigated by Elhefnawy [5], where the stability analysis has been made of a basic flow of streaming fluids in the presence of an oblique periodic electric field.

Zakaria et al [6] have analyzed the stability properties of periodic superposed magnetic fluids streaming through porous media under the influence of an oblique alternating magnetic field, where the system is composed of a middle fluid sheet of

${ }^{*}$ Received: August 14, 2010; accepted (in revised version): December 28, 2010. Communicated by Pingwen Zhang.

${ }^{\dagger}$ Department of Mathematics, Faculty of Science, Tanta University, Tanta, Egypt (kadry0zakaria@yahoo.com).

${ }^{\ddagger}$ Department of Mathematics, Faculty of Science, Tanta University, Tanta, Egypt (magdysirwah@yahoo.com).

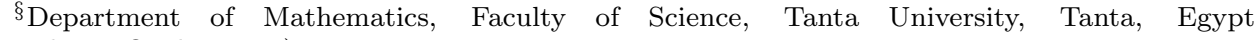
(sameh7977@yahoo.com). 
finite thickness embedded between two other bounded layers. Also, Zakaria et al [7] have investigated the instability properties of superposed conducting fluids streaming through porous media under the influence of a uniform magnetic field, where the system is composed of a middle fluid sheet of finite thickness embedded between two semi-infinite fluids. They found that the increase of the viscosity coefficient as well as the porosity plays an irregular role in the stability behavior, while the increase of the fluid velocity has a destabilizing influence in the stability criteria.

In all the works cited above, the fluids have been considered to be Newtonian (fluids such as water, air, ethanol, and benzene are Newtonian, while oil, liquid polymers, rubber, colloidal suspension and blood are non-Newtonian fluids). The study of non-Newtonian fluids is complicated compared with the Newtonian ones due to the interaction of the fluid viscosity and elasticity [8]. The mechanisms of non-Newtonian fluid sheets are of both practical and theoretical interest. In practice, it is often necessary to design atomization equipment for fluids which are highly non-Newtonian. From a theoretical point of view this problem is also of interest since a linear stability analysis of Newtonian fluids layers successfully predicts the characteristics of these layers under certain conditions. It is therefore tempting to investigate whether a linear stability analysis would lead to similar results for non-Newtonian fluids.

In recent years, there have been several studies $[9,10,11,12,13,14,15,16,17]$ on flows of non-Newtonian fluids, not only because of their technological significance, but also in view of the interesting mathematical features presented by the equations governing the flow. On the other hand, it is well known that the rheological properties of many fluids are not well modeled by the Navier-Stokes equations. For example, in most of these models, a significant reduction of the drag past solid walls has been observed. Moreover, elastic properties of real fluids can be detected and measured.

Liu et al [9] have studied the instability properties of two-dimensional nonNewtonian liquid sheets moving in an inviscid gaseous environment. They found that non-Newtonian liquid sheets have a higher growth rate than Newtonian liquid sheets for both symmetric and antisymmetric disturbances, indicating that non-Newtonian liquid sheets are more unstable than Newtonian liquid sheets.

Based on a modified Darcy's law for a viscoelastic fluid, Tan and Masuoka [13] extended Stokes first problem to that for an Oldroyd-B fluid in a porous half space, where an exact solution was obtained by using the Fourier sine transform. Khan et al [15] have produced analytical solutions for the magnetohydrodynamic flow of an Oldroyd-B fluid through a porous medium. They obtained the expressions for the velocity field and the tangential stress by means of the Fourier sine transform. Hayat et al [18] have discussed two Couette flows of a second grade fluid in a porous layer when (i) the bottom plate moves suddenly (ii) the bottom plate oscillates. They used a Laplace transform method to determine the analytic solutions. Kumar and Singh [19] have investigated the stability of a plane interface separating two viscoelastic (Rivlin-Ericksen) superposed fluids in the presence of suspended particles. They concluded that the system is stable for stable configuration and unstable for unstable configuration in the presence of suspended particles.

In this paper, we consider a system composed of a non-Newtonian (viscoelastic) fluid sheet of finite thickness embedded between two semi-infinite fluids. The system is influenced by an oblique magnetic field. The objective of the present work is to investigate the mechanisms of stability of three magnetic fluid layers in porous media, and to find whether a linear stability analysis for magnetic non-Newtonian fluids layers would result in conclusions similar to Newtonian fluids. The plan of this work is as 
follows: In next section, we will give a description of the problem including the basic equations of the fluid mechanics and Maxwell's equations governing the motion of our model. In the third section the liner stability analysis and the equilibrium solutions are derived. The fourth section and its subsections are concerned with the derivation of the characteristic equations and numerical applications for stability configuration. Also in this section the streamlines distribution are plotted and discussed. In the fifth section, the limiting case of the stability of one interface is investigated, where some stability diagrams are plotted and studied. Finally, the salient results of our analysis are discussed in the conclusion.

\section{Statement of the problem}

2.1. Governing equations. Consider an infinite horizontal viscoelastic fluid sheet of vertical height $2 a$ confined between two semi-infinite superposed viscoelastic fluids. Figure 2.1 represents a sketch of the system under consideration where the coordinates are chosen such that the $x$-axis is parallel to the direction of the fluid sheet flow and the $y$-axis is normal to the fluid sheet with its origin located at the middle plane of the fluid sheet. The lower fluid occupies the region $-a<y<-\infty$, the middle fluid is contained in the region typified by $-a<y<a$ while the range $a<y<$ $\infty$ represents the upper fluid. The system is considered to be influenced by the gravity force $\mathbf{g}(0, g)$ in the negative $y$-direction. The two interfaces between the fluids are assumed to be well defined, initially flat, and form the interfaces $y=-a$ and $y=a$. In fact, sharp interfaces between the fluids may not exist. Rather, there is an ill-defined transition region in which the two fluids intermix. The width of this transition zone is usually small compared with the other characteristic length of the motion; hence, for the purpose of the mathematical analysis, we shall assume that the fluids are separated by sharp interfaces. The two interfaces are parallel and the flow in each phase are everywhere parallel to each other. The surface deflections are expressed by $y=\xi_{1}(x, t)$ at $y=-a$ and $y=\xi_{2}(x, t)$ at $y=a$, where $y= \pm a$ are the equilibrium positions of the two interfaces, i.e. the positions without disturbances, and $\xi_{l},(l=1,2)$ is the displacement of a point on the surface (the size of the disturbance at a point). The fluids are initially assumed to be stressed by an oblique uniform magnetic field

$$
\mathbf{H}^{(j)}=H^{(j)}\left(\cos \left(\theta_{j}\right) \mathbf{e}_{x}+\sin \left(\theta_{j}\right) \mathbf{e}_{y}\right), \quad j=1,2,3,
$$

where $H^{(j)}$ is the amplitude of the field, and $\theta_{j} \in[0, \pi]$ represents the angle between the field $\mathbf{H}^{(j)}$ and the $x$-axis. The index $j=1,2$ and 3 distinguishs the quantities in the lower fluid, plane sheet and upper phase respectively. The unit vectors $\mathbf{e}_{x}$ and $\mathbf{e}_{y}$ are in the $x$ - and $y$ - directions.

The fundamental equation that governs and describes the behavior of fluid bulk through a porous media can be written as $[15,18,20]$

$$
\rho_{j} \frac{d \mathbf{u}^{(j)}}{d t}=-\nabla \bar{p}^{(j)}+\nabla \cdot \mathbf{S}^{(j)}+\mathbf{R}^{(j)} .
$$

Here $d / d t \equiv \partial / \partial t+\left(\mathbf{u}^{(j)} \cdot \nabla\right)$ stands for the convective derivative, $\partial / \partial t$ is the partial derivative with respect to the time $t, \nabla \equiv(\partial / \partial x, \partial / \partial y)$ denotes the horizontal gradient operator, $\mathbf{u}^{(j)}$ is the velocity vector, $\bar{p}^{(j)}=p^{(j)}+\rho_{j} g y$ indicates the total hydrostatic pressure, and $\mathbf{R}^{(j)}$ is the Darcy resistance for the Oldroyd-B fluid in a porous medium.

The stress tensor $\mathbf{S}^{(j)}$ is described by the Oldroyd-B constitutive equation given 


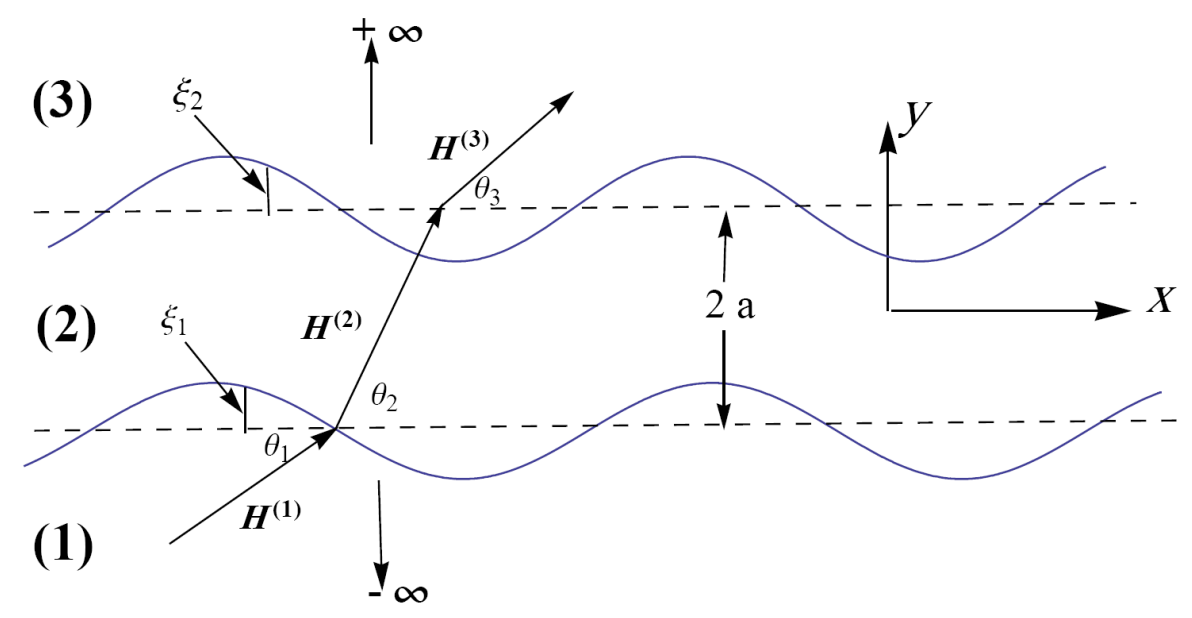

FIG. 2.1. Sketch of the system under consideration.

by $[8,13,17]$

$$
\left(1+\lambda_{1}^{(j)} \frac{\partial}{\partial t}\right) \mathbf{S}^{(j)}=2 \mu_{j}\left(1+\lambda_{2}^{(j)} \frac{\partial}{\partial t}\right) \mathbf{E}^{(j)}
$$

where $\mathbf{E}^{(j)}$ is the strain tensor of the form

$$
\mathbf{E}^{(j)}=\frac{1}{2}\left[\nabla \mathbf{u}^{(j)}+\left(\nabla \mathbf{u}^{(j)}\right)^{T}\right]
$$

where $\mu_{j}$ is the dynamic viscosity, $\lambda_{1}^{(j)}$ denotes the characteristic relaxation time depending on viscoelasticity and $\lambda_{2}^{(j)}$ is the constant deformation retardation time. It is assumed that $\lambda_{1}^{(j)} \geq \lambda_{2}^{(j)} \geq 0$ (see [13]). The superscript $T$ indicates the matrix transpose.

By analogy with the Oldroyd-B model, the following phenomenological model, which relates the pressure drop and velocity for a viscoelastic fluid in an unbounded porous medium, has been introduced [13, 21]:

$$
\left(1+\lambda_{1}^{(j)} \frac{\partial}{\partial t}\right) \nabla p^{(j)}=-\frac{\mu_{j} \phi_{j}}{q}\left(1+\lambda_{2}^{(j)} \frac{\partial}{\partial t}\right) \mathbf{u}^{(j)},
$$

where $\phi$ denotes the porosity and $q$ is the permeability of the porous media. In general, permeability is a tensor which depends on the porous medium microstructure (shape, size, and orientation of pores), but in the case of isotropy it reduces to a scalar; furthermore, when the Reynolds number is small, it is independent of the flow rate and the fluid properties. On the other hand, we apply the Brinkman approximation $[17,22,23]$, in which the fluid viscosity given by Equation (2.3) and the effective viscosity of the porous media in Equation (2.5) are equal to each other. The above equation shows that when $\lambda_{1}^{(j)}=\lambda_{2}^{(j)}=0$, the modified Darcy's law can be simplified to Darcy's law. 
The relation between the flow resistance $\mathbf{R}^{(j)}$ and velocity $\mathbf{u}^{(j)}$ can be inferred from Equation (2.5) to satisfy the following Equation:

$$
\left(1+\lambda_{1}^{(j)} \frac{\partial}{\partial t}\right) \mathbf{R}^{(j)}=-\frac{\mu_{j} \phi_{j}}{q_{j}}\left(1+\lambda_{2}^{(j)} \frac{\partial}{\partial t}\right) \mathbf{u}^{(j)} .
$$

It is useful at this point to nondimensionalize the governing equations and the boundary conditions. In fact, we will use dimensionless variables to provide improved insight into the physics and in order to better understand hydrodynamic stability. We shall use an asterisk as a superscript to the dimensional form and omit the asterisk for the dimensionless form where it is desirable to use the forms of the same physical quantities. Thus, we may henceforth write $u^{*}\left(x^{*}, t^{*}\right)$ for the dimensional and $u(x, t)$ for the dimensionless total velocity of a disturbed flow, since we define the corresponding dimensionless variables using the half thickness of the middle fluid sheet $a$ : the stream velocity $u^{*}=\sqrt{a g} u$, the time $t^{*}=\sqrt{a / g} t$, the pressure $p^{*}=a g \rho_{2} p$, the stream function $\psi^{*}=\sqrt{a^{3} g} \psi$, and $\left(x^{*}, y^{*}\right)=a(x, y)$. The applied magnetic field and the magnetic potential are made dimensionless by $H^{*}=\sqrt{a g \rho_{2} / \mu_{e 2}} H$ and $\chi^{*}=\sqrt{a^{3} g \rho_{2} / \mu_{e 2}} \chi$ respectively.

Assuming a quiescent initial state, the base state velocity in the fluid layers in which the flow is steady and fully developed is zero. Thus in the dimensionless form Equation (2.2) along with Equation (2.3), (2.6) yields

$$
\begin{aligned}
& \hat{\rho}_{j}\left(1+\lambda_{1}^{(j)} \frac{\partial}{\partial t}\right) \frac{\partial \mathbf{u}^{(j)}}{\partial t} \\
= & -\left(1+\lambda_{1}^{(j)} \frac{\partial}{\partial t}\right) \nabla \bar{p}^{(j)}+\frac{\hat{\mu}_{j}}{R e_{2}}\left(1+\lambda_{2}^{(j)} \frac{\partial}{\partial t}\right)\left\{2 \nabla \cdot \mathbf{E}^{(j)}+\frac{\mathbf{u}^{(j)}}{Q_{j}}\right\},
\end{aligned}
$$

associated with the continuity equation which expresses the conservation of mass:

$$
\nabla \cdot \mathbf{u}^{(j)}=0
$$

where $\hat{\rho}_{j}=\rho_{j} / \rho_{2}$ is the fluid density ratio, the parameter $\hat{\mu}_{j}=\mu_{j} / \mu_{2}$ represents the ratio of the dynamic viscosities, $R e_{2}=\rho_{2} \sqrt{a^{3} g} / \mu_{2}$ denotes the Reynolds number of the middle layer, and $Q_{j}=\frac{q_{j} / a}{\phi_{j}}$ is the permeability parameter. Note that Equation (2.7) can be simplified to the empirical modified Darcy's law Equation (2.5) if the unsteady term $\partial \mathbf{u}^{(j)} / \partial t$ and the viscous term $\nabla . \mathbf{E}^{(j)}$ are ignored.

We shall assume that there are no free currents at the surface of separation in the equilibrium state, and hence, in the magneto quasi-static system with negligible displacement current, Maxwell's equations are reduced to Gauss's and Ampér's laws $[24,25]$, which are

$$
\begin{aligned}
& \nabla \cdot \mathbf{B}^{(j)}=0, \\
& \nabla \times \mathbf{h}^{(j)}=0,
\end{aligned}
$$

where, the notation $\times$ refers to the vector product of two vectors, $\mathbf{B}^{(j)}=\mu_{e j} \mathbf{h}^{(j)}$ is the magnetic induction vector, $\mu_{e j}$ is the magnetic permeability, and $\mathbf{h}^{(j)}$ is the total magnetic field.

Equation (2.9) and (2.10) show that there exists a magnetic scalar potential $\chi^{(j)}(x, y, t)$ in each of the regions occupied by the fluids such that

$$
\mathbf{h}^{(j)}=\mathbf{H}^{(j)}-\nabla \chi^{(j)} .
$$


Therefore the magnetic scalar potential satisfies Laplace's equation

$$
\nabla^{2} \chi^{(j)}(x, y, t)=0
$$

2.2. Boundary conditions. In order to complete the formulation of the problem, the boundary conditions have to be specified. At the boundaries, the fluids and the magnetic stresses must be balanced. The components of these stresses consist of the hydrodynamics pressure, surface tension, porosity effects, and magnetic stresses [25]. The boundary conditions represented here are prescribed at the interface $y=\xi_{l}(x, t)$, where $\xi_{l}$ is the height of the disturbed interfaces away from its initial position $(y= \pm 1)$ which is defined in the next section. As the interface is deformed, all variables are slightly perturbed from their equilibrium values. Because the interfacial displacement is small, the boundary conditions on the perturbation interfacial variables need to be evaluated at the equilibrium position rather than at the interface. Therefore, it is necessary to express all the physical quantities involved in terms of Taylor series about $y= \pm 1$.

(i) Kinematics boundary conditions:

The flow field solutions of the above governing equations have to satisfy the kinematic and dynamic boundary conditions at the two interfaces, which can be taken as $y \approx \pm 1$ (the first order approximation for a small displacement of the interfaces due to the disturbance).

1- The normal component of the velocity vector in each of the phases of the system is continuous on the dividing surface. This implies that

$$
\mathbf{n}_{l} \cdot \mathbf{u}^{(l)}=\mathbf{n}_{l} \cdot \mathbf{u}^{(l+1)}, \quad y=(-1)^{l}, \quad l=1,2,
$$

where $\mathbf{n}_{l}$ is the outward normal unit vector to the interfaces which is given from the relation $\mathbf{n}_{l}=\nabla F_{l} /\left|\nabla F_{l}\right|$, and $F_{l}(x, y, t)$ is the surface geometry defined by $F_{l}=$ $y-\xi_{l}(x, t)= \pm 1$.

2- Since the interfaces are moving with the fluids $\left(D F_{l} / D t=0\right)$, we require that

$$
u_{y}^{l,(l+1)}+\frac{\partial \xi_{l}}{\partial t}=0, \quad y=(-1)^{l}, \quad l=1,2 .
$$

3- The jump in the shearing stresses must be zero across the interfaces, so that

$$
S_{x y}=0 \text { or }\left|\left[\hat{\mu}_{l}(\omega)\left(\frac{\partial u_{x}^{(l)}}{\partial y}+\frac{\partial u_{y}^{(l)}}{\partial x}\right)\right]\right|_{l}^{l+1}=0, \quad y=(-1)^{l}, \quad l=1,2,
$$

where, $\left(u_{x}, u_{y}\right)$ are the velocity components due to disturbances and $\hat{\mu}_{l}(\omega)$ is the frequency-dependent viscosity ratio defined in the following section. The notation $|[X]|$ is used here to signify the difference in some quantity $X$ across the interfaces.

(ii) Maxwell's conditions, for the magnetic potential

We use Maxwell's conditions on the magnetic field where no free surface charges are present on the interfaces as follows:

1- The continuity of the normal component of the magnetic displacement at the interfaces gives

$$
\mathbf{n}_{l} . \hat{\mu}_{e l} \mathbf{H}^{(l)}=\mathbf{n}_{l} . \mathbf{H}^{(l+1)}, \quad y=(-1)^{l}, \quad l=1,2,
$$


for which the zero order term reads

$$
\hat{\mu}_{e l} H^{(l)} \sin \left(\theta_{l}\right)=H^{(l+1)} \sin \left(\theta_{l+l}\right),
$$

where $\hat{\mu}_{e 1}=\mu_{e 1} / \mu_{e 2}$ and $\hat{\mu}_{e 2}=\mu_{e 3} / \mu_{e 2}$ are the magnetic permeability ratios. Using the zero order term to express both $H^{(2)}, H^{(3)}$, and $\theta_{2}, \theta_{3}$ in terms of $H^{(1)}$ and $\theta_{1}$ respectively, the first order reads

$$
\begin{gathered}
\frac{\partial \chi^{(2)}}{\partial y}-\hat{\mu}_{e 1} \frac{\partial \chi^{(1)}}{\partial y}=\left(\hat{\mu}_{e 1}-1\right) H^{(1)} \cos \left(\theta_{1}\right) \frac{\partial \xi_{1}}{\partial x}, \quad y=-1, \\
\frac{\partial \chi^{(2)}}{\partial y}-\hat{\mu}_{e 2} \frac{\partial \chi^{(3)}}{\partial y}=\left(\hat{\mu}_{e 2}-1\right) H^{(1)} \cos \left(\theta_{1}\right) \frac{\partial \xi_{2}}{\partial x}, \quad y=1 .
\end{gathered}
$$

2- The tangential component of the magnetic field is zero across the interfaces, so that

$$
\mathbf{n}_{l} \times \mathbf{H}^{(l)}=\mathbf{n}_{l} \times \mathbf{H}^{(l+1)}, \quad y=(-1)^{l}, \quad l=1,2 .
$$

From this equation, the zero order term reads

$$
H^{(l)} \cos \left(\theta_{l}\right)=H^{(l+1)} \cos \left(\theta_{l+l}\right),
$$

and the first order term has the form

$$
\begin{gathered}
\frac{\partial \chi^{(2)}}{\partial x}-\frac{\partial \chi^{(1)}}{\partial x}=\left(\hat{\mu}_{e 2}-1\right) H^{(1)} \sin \left(\theta_{1}\right) \frac{\partial \xi_{1}}{\partial x}, \quad y=-1, \\
\frac{\partial \chi^{(3)}}{\partial x}-\frac{\partial \chi^{(2)}}{\partial x}=\left(\hat{\mu}_{e 1} / \hat{\mu}_{e 2}-\hat{\mu}_{e 1}\right) H^{(1)} \sin \left(\theta_{1}\right) \frac{\partial \xi_{2}}{\partial x}, \quad y=1 .
\end{gathered}
$$

(iii) Component of the normal stress tensor

The normal component of the stress tensor is discontinuous by the amount of the surface tension [6]. Thus, the balance at the dividing surfaces gives (the dynamical boundary conditions)

$$
\left|\left[\mathbf{n}_{l} \cdot \mathbf{S} \cdot \mathbf{n}_{l}\right]\right|_{l}^{l+1}=W_{l} \nabla \cdot \mathbf{n}_{l}, \quad y=(-1)^{l}, \quad l=1,2 .
$$

Here, $W_{l}=T_{l} / a^{2} g \rho_{2}$ is the dimensionless Weber number, where $T_{l}$ represents the interfacial surface tension coefficient, and $\mathbf{S}$ is the total stress tensor at the interfaces given by

$$
S_{m n}=-p \delta_{m n}+\mu(\omega)\left(\frac{\partial u_{m}}{\partial x_{n}}+\frac{\partial u_{n}}{\partial x_{m}}\right)+\mu_{e} H_{m} H_{n}-\frac{1}{2} \mu_{e} H^{2} \delta_{m n},
$$

where $\delta_{m n}$ is the Kronecker's delta symbol which equals 1 if $m=n$ and 0 otherwise.

Substituting Equation (2.19) into Equation (2.18) yields

$$
\left|\left[-p+2 \mu(\omega) \frac{\partial u_{y}}{\partial y}+\mu_{e}\left(H_{n}^{2}-\frac{1}{2} H^{2}\right)\right]\right|_{l}^{l+1}=W_{l} \nabla \cdot \mathbf{n}_{l} .
$$

\section{Stability analysis}


3.1. Linear perturbation. In order to investigate the stability of the present problem, the interfaces between the fluids will be assumed to be perturbed about their equilibrium location and will cause a displacement of the material particles of the fluid system. This displacement may be described by the equation

$$
\xi_{l}(x, t)=\hat{\xi}_{l} e^{i k x+\omega t}+c . c, \quad l=1,2,
$$

where $\hat{\xi}_{l}$ is the initial amplitude of the disturbance, which is taken to be much smaller than the half-thickness $a$ of the middle sheet, $k$ is the wave number of the disturbance, which is assumed to be real and positive $(k=2 \pi / \lambda$, where $\lambda$ is the wavelength of the disturbance), $\omega$ is a complex frequency $\left(\omega=\omega_{r}+i \omega_{i}\right.$, where $\omega_{r}$ represents the rate of growth of the disturbance, $\omega_{i}$ is $2 \pi$ times the disturbance frequency), the symbol $i$ denotes $\sqrt{-1}$, the imaginary number and c.c represents the complex conjugate of the preceding terms.

The deformation in the interfaces $y= \pm 1$ is due to the perturbation about the equilibrium values of all the other variables. The form of horizontal variation for all the other perturbed variables will be the same as the displacement description (3.1). In accordance with the interface deflection given by (3.1) and in view of a standard Fourier decomposition, we may similarly assume that the bulk solutions are periodic functions in $x$ and exponential functions in $t$, which are regarded as

$$
\left(\begin{array}{c}
\mathbf{S} \\
\mathbf{E} \\
\mathbf{u} \\
\chi \\
p
\end{array}\right)=\left(\begin{array}{c}
\hat{\mathbf{S}}(y) \\
\hat{\mathbf{E}}(y) \\
\hat{\mathbf{u}}(y) \\
\hat{\chi}(y) \\
\hat{p}(y)
\end{array}\right) e^{i k x+\omega t+c . c .}
$$

Substituting Equation (2.4) and (3.2) into Equation (2.3) yields

$$
\mathbf{S}^{(j)}=2 \mu_{j}(\omega) \mathbf{E}^{(j)},
$$

where

$$
\mu_{j}(\omega)=\mu_{j} \frac{1+\lambda_{2}^{(j)} \omega}{1+\lambda_{1}^{(j)} \omega} .
$$

It should be noted that this model is sometimes called Maxwell-Jeffreys fluid and includes, as special cases, the Maxwell model at $\lambda_{2}^{(j)}=0$, and when $\lambda_{1}^{(j)}=\lambda_{2}^{(j)}=0$, the sheet of viscoelastic fluid transfors into a Newtonian one. Thus, in this linearized stability analysis about the static base state, the only way in which viscoelasticity appears in the calculation is through the growth-rate dependence of viscosity. In principle, one could therefore obtain the dispersion relation relating the growth rate to the wave number by simply substituting the frequency-dependent viscosity $\mu_{j}(\omega)$ instead of the constant viscosity in the characteristic equation for a Newtonian fluid.

3.2. Lines of solutions. The equations of motion and the boundary conditions mentioned previously will be solved under the assumption that the perturbations are small; so, all equations of motion and boundary conditions will be linearized in the perturbed quantities. Eliminating the pressure term by taking the rotation of Equation (2.7), using (2.8) we get the equation

$$
\left\{\hat{\rho}_{j} \omega+\frac{\hat{\mu}_{j}(\omega)}{Q_{j} R e_{2}}\right\} \nabla \times \mathbf{u}^{(j)}=\frac{2 \hat{\mu}_{j}(\omega)}{R e_{2}} \nabla \times \nabla . \mathbf{E}^{(j)},
$$


since we define the divergence of the tensor as [24]

$$
\nabla . \mathbf{E}=\mathbf{e}_{\kappa} \frac{\partial}{\partial x_{\kappa}}\left(E_{m n} \mathbf{e}_{m} \mathbf{e}_{n}\right)=\mathbf{e}_{n} \frac{\partial E_{m n}}{\partial x_{m}}, \quad m, n, \kappa \in\{x, y\} .
$$

The solution of the above system of governing equations and boundary conditions can be facilitated by defining a stream function $\psi$ of the time and space coordinates, which automatically satisfies Equation (2.8), where

$$
u_{x}=\frac{\partial \psi}{\partial y}, u_{y}=-\frac{\partial \psi}{\partial x} .
$$

Using the normal mode approach we write the perturbations in the form

$$
\psi=\hat{\psi}(y) e^{i k x+\omega t}+\text { c.c. }
$$

Substituting Equation (3.6) into Equation (3.4) (taking into account Equation (3.5)), we obtain the equation

$$
\nabla^{4} \psi^{(j)}-\left(L_{j}^{2}-k^{2}\right) \nabla^{2} \psi^{(j)}=0
$$

where

$$
L_{j}=\sqrt{k^{2}+\frac{\hat{\rho}_{j} R e_{2} \omega}{\hat{\mu}_{j}(\omega)}+\frac{1}{Q_{j}}} .
$$

Using the solution (3.7), Equation (3.8) can be transformed into the following form:

$$
\frac{d^{4} \hat{\psi}^{(j)}}{d y^{4}}-\left(L_{j}^{2}+k^{2}\right) \frac{d^{2} \hat{\psi}^{(j)}}{d y^{2}}+k^{2} L_{j}^{2} \hat{\psi}^{(j)}=0 .
$$

It is obvious that the analytical solution of Equation (3.9) is of the form

$$
\hat{\psi}^{(j)}(y)=A_{1}^{(j)} e^{k y}+A_{2}^{(j)} e^{-k y}+A_{3}^{(j)} e^{L_{j} y}+A_{4}^{(j)} e^{-L_{j} y} .
$$

Since the boundary conditions require that the disturbances vanish as $y \rightarrow \pm \infty$ (i.e. $A_{2}^{(1)}=A_{4}^{(1)}=A_{1}^{(3)}=A_{3}^{(3)}=0$ ), we have the stream function in the three layers:

$$
\begin{aligned}
& \psi^{(1)}=\left(A_{1}^{(1)} e^{k y}+A_{3}^{(1)} e^{L_{1} y}\right) e^{i k x+\omega t}+\text { c.c }, \quad y<-1, \\
& \psi^{(2)}=\left(A_{1}^{(2)} e^{k y}+A_{2}^{(2)} e^{-k y}+A_{3}^{(2)} e^{L_{2} y}+A_{4}^{(2)} e^{-L_{2} y}\right) e^{i k x+\omega t}+c . c, \quad-1<y<1 \text {, } \\
& \psi^{(3)}=\left(A_{2}^{(3)} e^{-k y}+A_{4}^{(3)} e^{-L_{3} y}\right) e^{i k x+\omega t}+\text { c.c, } \quad y>1 \text {. }
\end{aligned}
$$

The coefficients $A$ 's can be determined from the above boundary conditions, where

$$
\begin{aligned}
& A_{1}^{(1)}=A_{11}^{(1)} \hat{\xi}_{1}+A_{12}^{(1)} \hat{\xi}_{2}, \quad A_{3}^{(1)}=A_{31}^{(1)} \hat{\xi}_{1}+A_{32}^{(1)} \hat{\xi}_{2}, \\
& A_{1}^{(2)}=A_{11}^{(2)} \hat{\xi}_{1}+A_{12}^{(2)} \hat{\xi}_{2}, A_{2}^{(2)}=A_{21}^{(2)} \hat{\xi}_{1}+A_{22}^{(2)} \hat{\xi}_{2} \text {, } \\
& A_{3}^{(2)}=A_{31}^{(2)} \hat{\xi}_{1}+A_{32}^{(2)} \hat{\xi}_{2}, A_{4}^{(2)}=A_{41}^{(2)} \hat{\xi}_{1}+A_{42}^{(2)} \hat{\xi}_{2} \text {, } \\
& A_{2}^{(3)}=A_{21}^{(3)} \hat{\xi}_{1}+A_{22}^{(3)} \hat{\xi}_{2}, A_{4}^{(3)}=A_{41}^{(3)} \hat{\xi}_{1}+A_{42}^{(3)} \hat{\xi}_{2} \text {, }
\end{aligned}
$$

and the values $A_{q l}^{(j)},(q=1, \ldots, 4)$ are given in Appendix A. 
To determine the pressure, Equation (3.2) along with $(2.7,3.3)$ yields

$$
p^{(j)}=\frac{1}{i k}\left\{\frac{\hat{\mu}_{j}(\omega)}{R e_{2}}\left[\frac{\partial^{3} \psi^{(j)}}{\partial y^{3}}+\frac{\partial^{3} \psi^{(j)}}{\partial x^{2} \partial y}-\frac{1}{Q_{j}} \frac{\partial \psi^{(j)}}{\partial y}\right]-\hat{\rho}_{j} \frac{\partial^{2} \psi^{(j)}}{\partial x \partial t}\right\} .
$$

The solution of the magnetic potential, in view of Equation (2.12) and (3.2), may be taken to be of the form

$$
\begin{aligned}
& \chi^{(1)}=B_{1}^{(1)} e^{i k x+k y+\omega t}+c . c, \quad y<-1, \\
& \chi^{(2)}=\left(B_{1}^{(2)} e^{k y}+B_{2}^{(2)} e^{-k y}\right) e^{i k x+\omega t}+c . c, \quad-1<y<1, \\
& \chi^{(3)}=B_{2}^{(3)} e^{i k x-k y+\omega t}+c . c, \quad y>1 .
\end{aligned}
$$

The coefficients $B$ 's are determined from the boundary conditions, where

$$
\begin{aligned}
& B_{1}^{(1)}=B_{11}^{(1)} \hat{\xi}_{1}+B_{12}^{(1)} \hat{\xi}_{2}, \quad B_{1}^{(2)}=B_{11}^{(2)} \hat{\xi}_{1}+B_{12}^{(2)} \hat{\xi}_{2}, \\
& B_{2}^{(2)}=B_{21}^{(2)} \hat{\xi}_{1}+B_{22}^{(2)} \hat{\xi}_{2}, \quad B_{2}^{(3)}=B_{21}^{(3)} \hat{\xi}_{1}+B_{22}^{(3)} \hat{\xi}_{2},
\end{aligned}
$$

and the algebraic expressions of the coefficients $B_{p l}^{(r)},(r, p, l=1,2)$ are defined in Appendix B.

4. Derivation of the characteristic equations and their stability

In this section, we will determine the boundary-value problem cited above, in which we obtain the characteristic equation governing the interfacial waves. The components of these stresses consist of hydrodynamic pressure, porosity effects, surface tension stresses, and magnetic stresses, and must be balanced at the boundaries among the fluids. Inserting Equation (3.11-3.15) into the dynamical conditions (2.20), we get a linear system of homogenous algebraic equations in terms of $\hat{\xi}_{1}$ and $\hat{\xi}_{2}$. This homogeneous system of equations can be written in matrix form as

$$
\mathbf{A X}=\mathbf{0},
$$

where matrix $\mathbf{A}$ and $\mathbf{X}$ take the following form:

$$
\mathbf{A}=\left(\begin{array}{cc}
\Omega_{11} & \Omega_{12} \\
\Omega_{21} & \Omega_{22}
\end{array}\right), \quad \mathbf{X}=\left(\begin{array}{c}
\hat{\xi}_{1} \\
\hat{\xi}_{2}
\end{array}\right) .
$$

Here, the first row in matrix $\mathbf{A}$ is given by

$$
\begin{aligned}
\Omega_{1 m}= & \frac{-1}{Q_{2} R e_{2}} \sum_{l=1}^{2} e^{(-1)^{l} k}\left\{\hat{\mu}_{2}(\omega)+Q_{2} R e_{2}\left[\omega+2 i(-1)^{l} k^{2} \hat{\mu}_{2}(\omega)\right]\right\} A_{l m}^{(2)} \\
& -\frac{\hat{\mu}_{1}(\omega)}{Q_{1} R e_{2}}\left\{e^{-k}\left[1-2 i k^{2} Q_{1} R e_{2}\right] A_{1 m}^{(1)}-2 i k L_{1} e^{-L_{1}} Q_{1} R e_{2} A_{3 m}^{(1)}\right\} \\
& +2 i k L_{2} e^{-L_{2}} \hat{\mu}_{2}(\omega)\left[e^{2 L_{2}} A_{4 m}^{(2)}-A_{3 m}^{(2)}\right]-\hat{\rho}_{1} \omega e^{-k} A_{1 m}^{(1)}-(2-m) \\
& \times\left(\hat{\rho}_{1}-k^{2} W_{1}-1\right)+f_{1 m}, \\
f_{1 m}= & k H^{(1)} e^{-k}\left\{\hat{\mu}_{e 1} \sin \left(\theta_{1}\right)\left(B_{1 m}^{(1)}-B_{1 m}^{(2)}+e^{2 k} B_{2 m}^{(2)}\right)+i \cos \left(\theta_{1}\right)\left(B_{1 m}^{(2)}-\hat{\mu}_{e 1} B_{1 m}^{(1)}\right.\right. \\
& \left.\left.+e^{2 k} B_{2 m}^{(2)}\right)\right\}
\end{aligned}
$$


and the second row is taken the form

$$
\begin{aligned}
\Omega_{2 m}= & \frac{-1}{Q_{2} R e_{2}} \sum_{l=1}^{2} e^{(-1)^{l+1} k}\left\{\hat{\mu}_{2}(\omega)+Q_{2} R e_{2}\left[\omega+2 i(-1)^{l} k^{2} \hat{\mu}_{2}(\omega)\right]\right\} A_{l m}^{(2)} \\
& +\frac{\hat{\mu}_{3}(\omega)}{Q_{3} R e_{2}}\left\{e^{-k}\left[1+2 i k^{2} Q_{3} R e_{2}\right] A_{2 m}^{(3)}+2 i k L_{3} e^{-L_{3}} Q_{3} R e_{2} A_{4 m}^{(3)}\right\} \\
& +2 i k L_{2} e^{-L_{2}} \hat{\mu}_{2}(\omega)\left[e^{2 L_{2}} A_{3 m}^{(2)}-A_{4 m}^{(2)}\right]+\hat{\rho}_{3} \omega e^{-k} A_{2 m}^{(3)}+(m-1) \\
& \times\left(\hat{\rho}_{3}+k^{2} W_{2}-1\right)+f_{2 m}, \\
f_{2 m}= & k H^{(1)} e^{-k}\left\{\hat{\mu}_{e 1} \sin \left(\theta_{1}\right)\left(B_{2 m}^{(3)}-B_{2 m}^{(2)}+e^{2 k} B_{1 m}^{(2)}\right)+i \cos \left(\theta_{1}\right)\left(B_{2 m}^{(2)}-\hat{\mu}_{e 2} B_{2 m}^{(3)}\right.\right. \\
& \left.\left.+e^{2 k} B_{1 m}^{(2)}\right)\right\} .
\end{aligned}
$$

For the nontrivial solutions of the unknown coefficients $\hat{\xi}_{1}$ and $\hat{\xi}_{2}$ of the system of equations, the determinant of the matrix $\mathbf{A}$ must be equal to zero, which gives the dispersion relation of the present problem. It should be noted that this yields a relation of the form

$$
|\mathbf{A}|=D(\omega, k)=0,
$$

which represents the linear dispersion equation for surface waves propagating through a non-Newtonian layer embedded between two other fluids with the influence of a constant oblique magnetic field. This dispersion relation describes the relationship between perturbation frequency $\omega$ and wavenumber $k$ for different parameters and controls the stability in the present problem. That is, each negative of the real part of $\omega$ corresponds to a stable mode of the interfacial disturbance. On the other hand, if the real part of $\omega$ is positive, the disturbance grows in time and the flow becomes unstable.

4.1. Special cases. It is clear that the eigenvalue relation (4.3) is somewhat more general and quite complex since $L_{j}$ involves square roots and so one can obtain other characteristic relations as limiting cases.

(i) For an inviscid fluid we get the characteristic equation as a special case of Equation (4.3) when $\mu_{j}=\lambda_{1}^{(j)}=\lambda_{2}^{(j)}=0$. Thus, by collecting the real and the imaginary terms in power order of $\omega$ with the help of symbolic computation software Mathematica, Equation (4.3) can be transformed into the form

$$
\omega^{4}+\left(\alpha_{11}+i \alpha_{12}\right) \omega^{3}+\left(\alpha_{21}+i \alpha_{22}\right) \omega^{2}+\left(\alpha_{31}+i \alpha_{32}\right) \omega+\alpha_{41}+i \alpha_{42}=0,
$$

where the coefficients $\alpha$ 's are clear from the context. Zakaria et al [6] obtained a similar equation in their study of temporal stability of an inviscid fluids in porous media. All the roots of Equation (4.4) will have negative real parts, and hence the corresponding system is stable, as the following conditions are satisfied [6, 26]:

$$
v_{1}>0, \quad v_{2}>0, \quad v_{3}>0, \quad v_{4} \geq 0,
$$

where, the values $v$ 's are given in Appendix C.

(ii) In the special case of a viscous fluid layer embedded in two viscous fluids (i.e. $\lambda_{1}^{(j)}=\lambda_{2}^{(j)}=0$ ), we obtain a characteristic equation not different from the general form of Equation (4.3), which is also an implicit dispersion relation between the growth rate and the wave number since the value $L_{j}$ is given by (3.8) reduced to $L_{j}=\sqrt{k^{2}+\frac{\hat{\rho}_{j} R e_{2} \omega}{\hat{\mu}_{j}}+\frac{1}{Q_{j}}}$. 


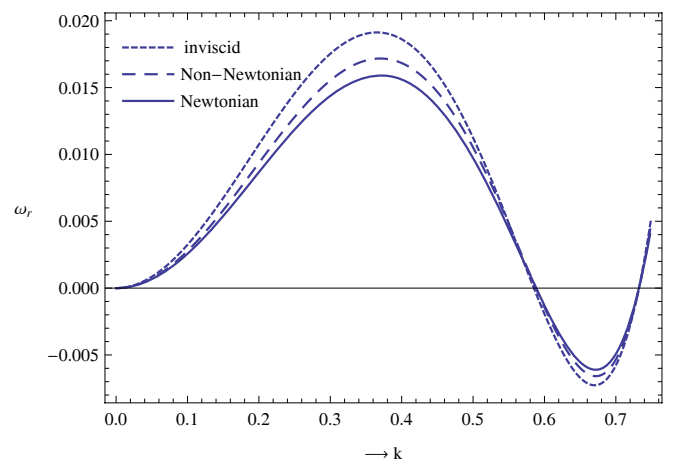

FIG. 4.1. Non-dimensional growth rate of different fluids versus non-dimensional wave number at $\hat{\rho}_{1}=0.5, \hat{\rho}_{3}=0.1, \hat{\mu}_{e 1}=2, \hat{\mu}_{e 2}=1, \hat{\mu}_{1}=2, \hat{\mu}_{3}=2, R e_{2}=800, Q_{1}=2, Q_{2}=5, Q_{3}=3, W_{1}=$ $2, W_{2}=1, \quad \lambda_{1}^{(1)}=0.4, \lambda_{2}^{(1)}=0.1, \lambda_{1}^{(2)}=0.5, \quad \lambda_{2}^{(2)}=0.8, \lambda_{1}^{(3)}=0.6, \lambda_{2}^{(3)}=0.3$.

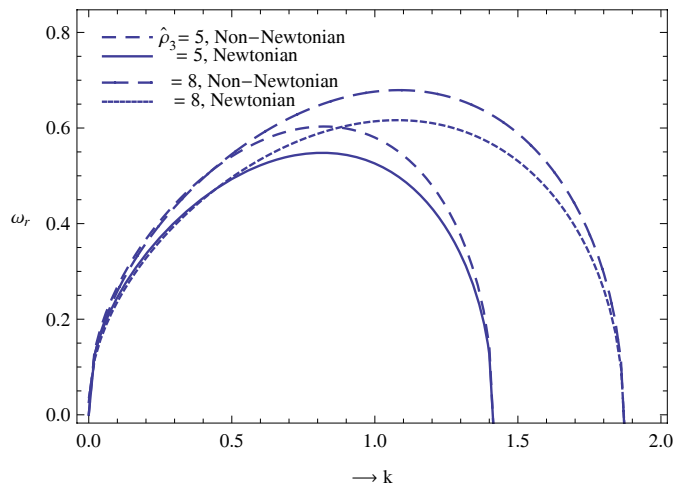

FIG. 4.2. Effects of the density ratio $\hat{\rho}_{3}$ in the plane $\left(\omega_{r}-k\right)$ at $H^{(1)}=5, \theta_{1}=0, \hat{\rho}_{1}=5, \hat{\mu}_{e 1}=$ $1, \hat{\mu}_{e 2}=2, \hat{\mu}_{1}=3, \hat{\mu}_{3}=4, R e_{2}=15, Q_{1}=0.1, Q_{2}=0.2, Q_{3}=0.3, W_{1}=2, W_{2}=1, \lambda_{1}^{(1)}=0.4, \lambda_{2}^{(1)}=$ $0.2, \lambda_{1}^{(2)}=0.5, \lambda_{2}^{(2)}=0.3, \lambda_{1}^{(3)}=0.6, \lambda_{2}^{(3)}=0.4$, on the wave growth rate of both Newtonian and nonNewtonian fluid layers with $\hat{\rho}_{3}=3,5$.

4.2. Applications. In order to discuss the stability diagrams, we first specify the parameters of Equation (4.3) which are used to control the stability behavior. These include the magnetic field, the magnetic permeability, the porosity effect, the density, the viscosity, and retardation and relaxation times. In the calculations given below all the physical parameters are sought in the dimensionless form as defined in Section 2.1. The stability of fluid sheets corresponds to negative values of the disturbance growth rate (i.e. $\omega_{r}<0$ ), and the disturbance growth rates of different fluids can be gained through solving the above corresponding dispersion relation numerically.

Figure 4.1 shows the variation of the non-dimensional growth rate $\omega_{r}$ with the non-dimensional wave number $k$ for a system having the parameters given in the caption of Figure 4.1. In this figure the continuous line represents the Newtonian fluid, the dotted curve denotes the inviscid fluid, and the dashed curve corresponds to the non-Newtonian fluid. It can be shown from Figure 4.1 that the maximum growth 


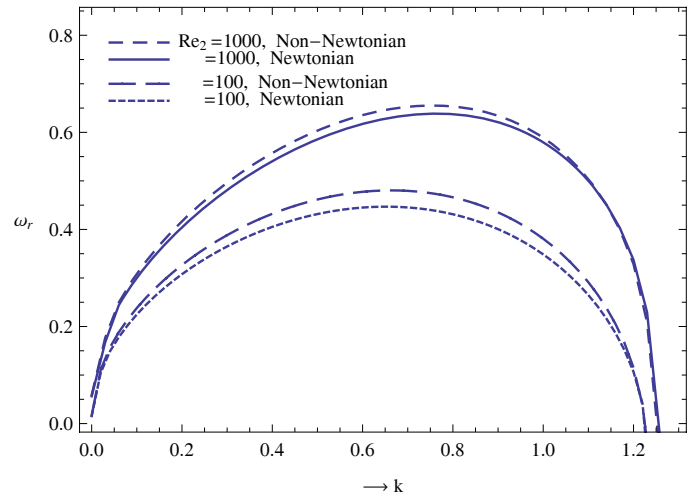

FIG. 4.3. Influence of the Reynolds number $R_{2}$ in the plane $\left(\omega_{r}-k\right)$ on the wave growth rate of both Newtonian and non-Newtonian fluid layers with $R_{2}=100,1000$ at the same system given in Figure 4.2 but with $\hat{\rho}_{3}=3$.

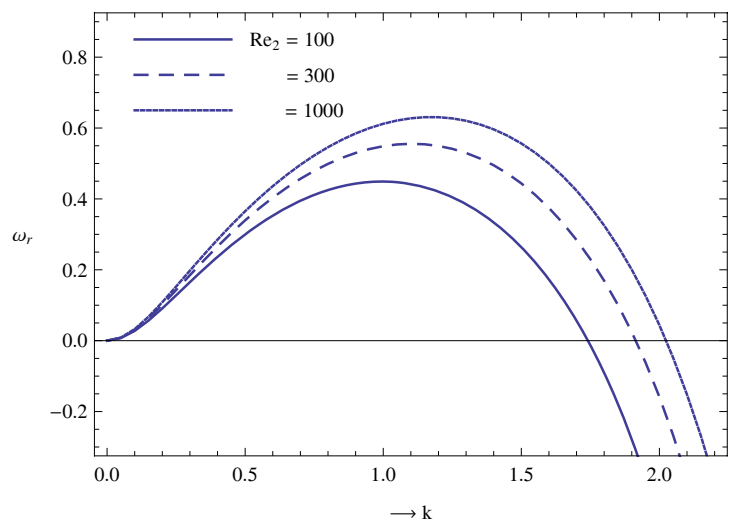

FIG. 4.4. Effects of the Reynolds number on non-dimensional growth rate of non-Newtonian fluids versus non-dimensional wave number at $H^{(1)}=0.1, \theta_{1}=0, \hat{\rho}_{1}=0.1, \hat{\rho}_{3}=0.15, \hat{\mu}_{e 1}=2, \hat{\mu}_{e 2}=$ $1, \hat{\mu}_{1}=2, \hat{\mu}_{3}=2, Q_{1}=2, Q_{2}=5, Q_{3}=3, W_{1}=2, W_{2}=1$, with Re $e_{2}=100$ (solid), 300 (dashed), 1000 (dotted).

rate for any non-Newtonian fluid lies above that corresponding to a Newtonian fluid and below that corresponding to an inviscid fluid. Similar results were reported by Liu et al [9] in their studies of the instability of two-dimensional non-Newtonian liquid sheets. The cutoff wave number (also called the critical wave number) can be defined as [9] the value of the wave number at the point where the growth rate curve crosses the wave number axis in the plots of wave growth rate versus wave number. In other words, the critical wave number is the value of the wave number which separates the stable motions from the unstable ones, and conversely can be obtained from the corresponding dispersion relations by setting $\omega_{r}=0$. Since the stability arises according to the negative sign of the real part of the complex frequency $\omega$, when the wave number is over the cutoff wave number the fluid sheet is stable. Therefore, it is concluded that, in the range of flow parameters associated with the 


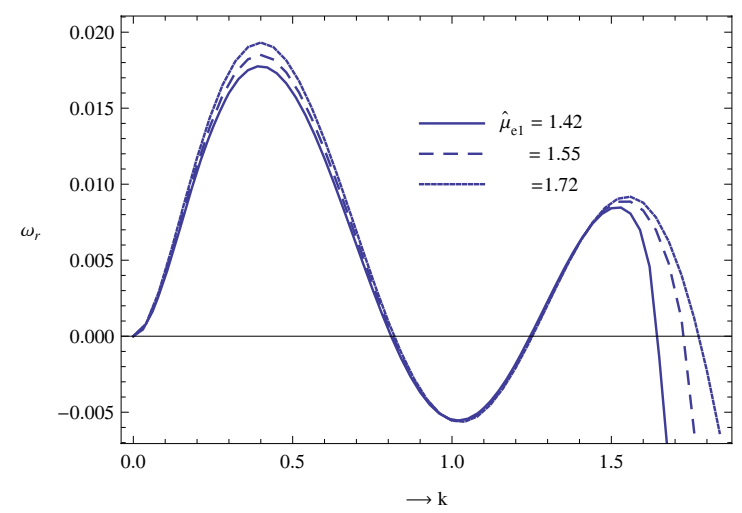

FIG. 4.5. The graph is constructed for $\omega_{r}$ versus $k$, for $H^{(1)}=3, \theta_{1}=0, \hat{\rho}_{1}=0.05, \hat{\rho}_{3}=$ $0.01, \quad \hat{\mu}_{1}=2, \quad \hat{\mu}_{3}=3, R e_{2}=1300, \quad Q_{1}=2, \quad Q_{2}=5, Q_{3}=3, W_{1}=2, W_{2}=1, \quad \lambda_{1}^{(1)}=0.5, \quad \lambda_{2}^{(1)}=$ $0.1, \lambda_{1}^{(2)}=0.5, \lambda_{2}^{(2)}=0.2, \lambda_{1}^{(3)}=0.5$, and $\lambda_{2}^{(3)}=0.1$, with $\hat{\mu}_{e 1}=1.42,1.55$, 1.72.

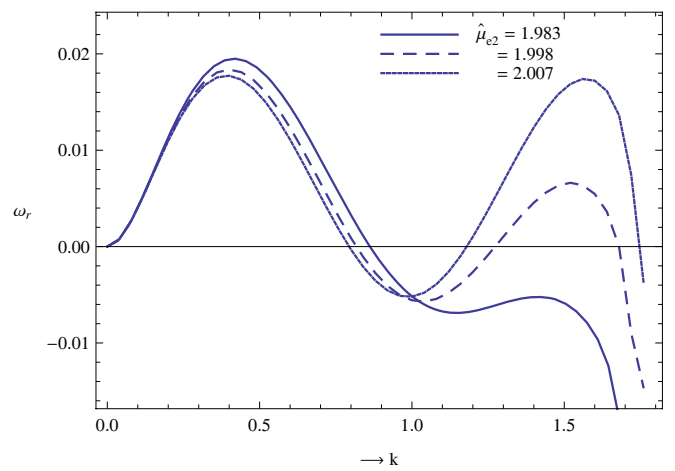

FIG. 4.6. The stability diagram in the plane $\left(\omega_{r}-k\right)$ for the same system given in Figure 4.5, with $\hat{\mu}_{e 1}=1.5$ at $\hat{\mu}_{e 2}=1.983$ (solid), 1.998 (dashed), 2.007 (dotted).

results of Figure 4.1, the region $0<k<0.58$ (the left side of the cutoff wave number) is unstable. Moreover, in this range, we observe that the sheet of such a non-Newtonian fluid is more unstable than a Newtonian fluid sheet. On the other hand the region $0.58<k<0.74$ (the negative values of the disturbance growth rate) is stable, and we notice that non-Newtonian fluid layer is more stable than a Newtonian fluid sheet.

The examination of the increase of the density ratio $\hat{\rho}_{3}\left(=\rho_{3} / \rho_{2}\right)$ on the wave growth rate of both Newtonian and non-Newtonian fluid layers is displayed in Figure 4.2. In this graph the solid line represents the Newtonian fluid, the dashed curve denotes the non-Newtonian fluid at the density ratio $\hat{\rho}_{3}=5$, while the dotted line and the dashed dotted curves denote respectively the Newtonian and the non-Newtonian fluids at the density ratio $\hat{\rho}_{3}=8$. It can be discovered from Figure 4.2 that when the fluid density ratio $\hat{\rho}_{3}=5$ is increased, both the growth rates and the cutoff wave number of Newtonian and non-Newtonian fluid layers increase. Thus we conclude that the increase of the density ratio $\hat{\rho}_{3}=5$ plays a destabilizing role in the stability behavior in the presence of the horizontal magnetic field. 


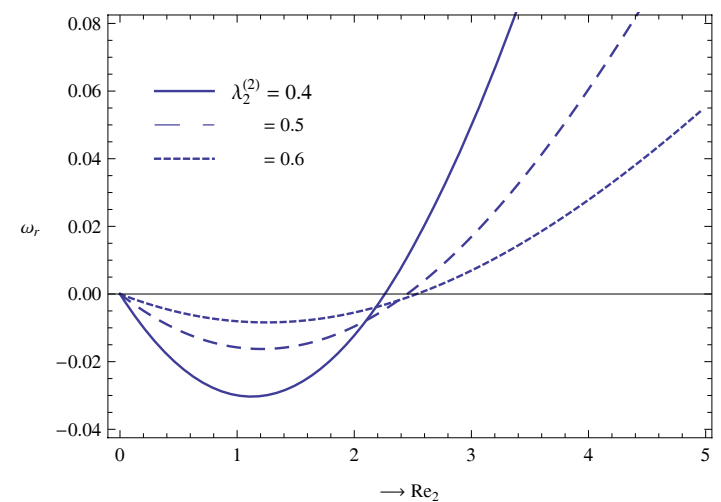

FIG. 4.7. Represents the stability diagrams in the plane $\left(\omega_{r}-\lambda_{1}^{(2)}\right)$ for a system having $H^{(1)}=$ $5.5, \theta_{1}=0, \hat{\mu}_{e 1}=0.2, \hat{\mu}_{e 2}=0.1, \quad \hat{\rho}_{1}=1.5, \hat{\rho}_{3}=3, \quad \hat{\mu}_{1}=0.2, \quad \hat{\mu}_{3}=0.4, R_{2}=200, Q_{1}=2, \quad Q_{2}=$ $5, Q_{3}=3, W_{1}=2, W_{2}=1, k=0.8, \lambda_{1}^{(1)}=0.5, \lambda_{2}^{(1)}=0.3, \quad \lambda_{1}^{(3)}=0.9$, and $\lambda_{2}^{(3)}=0.1$, at $\lambda_{2}^{(2)}=0.4$ (solid), 0.5 (dashed), 0.6 (dotted).

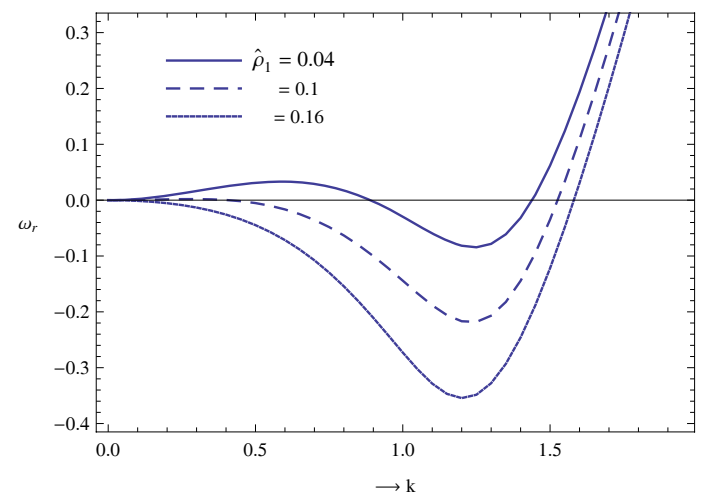

FIG. 4.8. The plane $\left(\omega_{r}-k\right)$ is shown for a system having $H^{(1)}=0.5, \theta_{1}=0, \hat{\mu}_{e 1}=2, \hat{\mu}_{e 2}=$ $1, \hat{\rho}_{3}=0.15, \hat{\mu}_{1}=2, \hat{\mu}_{3}=2, R e_{2}=1300, Q_{1}=2, Q_{2}=5, Q_{3}=3, W_{1}=2, W_{2}=1, \lambda_{1}^{(1)}=0.4, \lambda_{2}^{(1)}=$ $0.1, \lambda_{1}^{(2)}=0.5, \lambda_{2}^{(2)}=0.2, \lambda_{1}^{(3)}=0.5$, and $\lambda_{2}^{(3)}=0.1$, with $\hat{\rho}_{1}=0.04,0.1,0.16$.

In order to investigate the effect of the Reynolds number $R e_{2}$ on the stability criteria, numerical calculations for the dispersion relation (4.3) are made. The results of these calculations are displayed in Figure 4.3 in the plane $\left(\omega_{r}-k\right)$. The graph displayed in this plane is evaluated for the same system given in Figure 4.2, but with $\hat{\rho}_{3}=3$. In this graph the dotted line and the dashed dotted curves denote respectively the Newtonian and the non-Newtonian fluids at the Reynolds number $R e_{2}=100$, while the solid curve represents the Newtonian fluid and the dashed line corresponds to the non-Newtonian fluid at $R e_{2}=1000$. It can be seen from Figure 4.3 that when the Reynolds number is increased, the growth rate of Newtonian and non-Newtonian fluid layers increase. Thus we conclude that the increase of the Reynolds number plays a destabilizing role in the stability of the movement of the fluid.

Figure 4.4 is the plot of the wave number $k$ versus the growth rate $\omega_{r}$ for different 

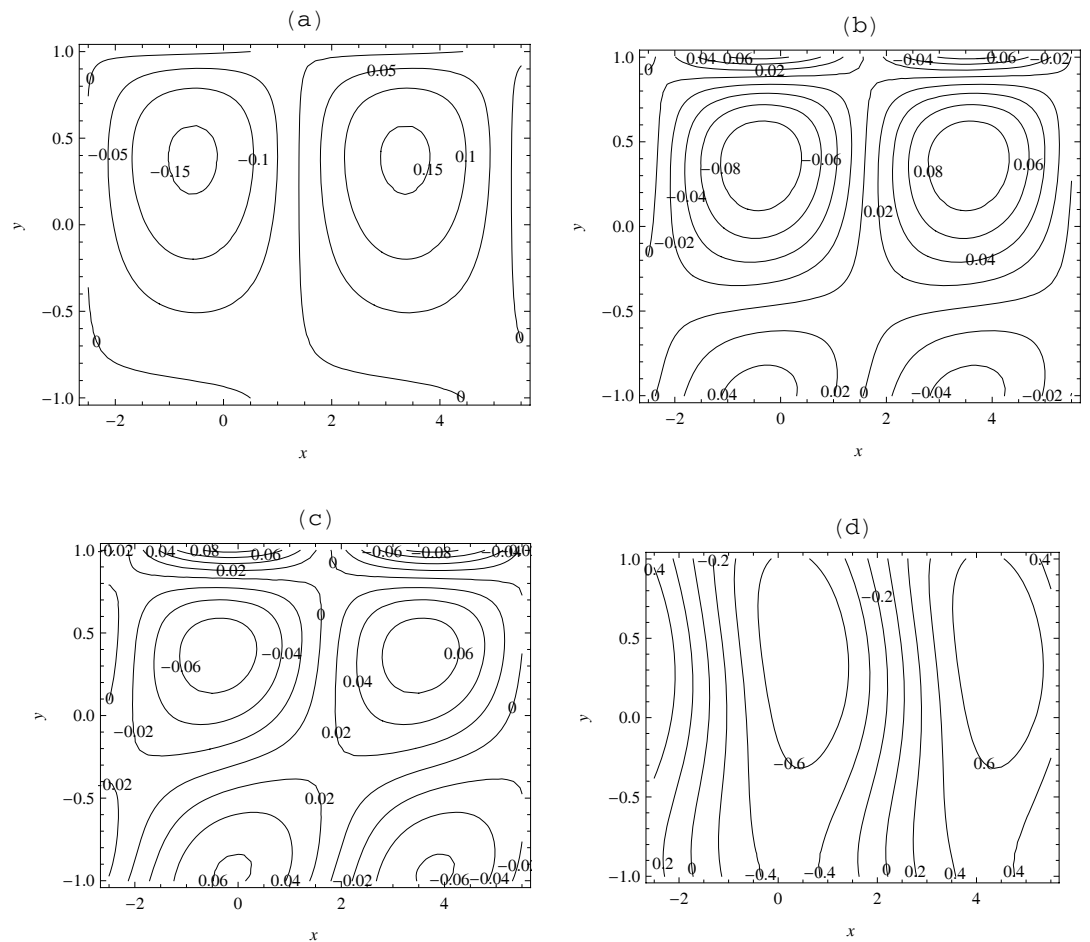

FIG. 4.9. Streamlines contours for a system having the same parameters considered in Figure 4.2, with, $\operatorname{Re}_{2}=500, k=0.8, t=0.1, \hat{\xi}_{1}=0.05, \hat{\xi}_{2}=0.07$. while $W_{2}=2,5,8$, and 10 of the parts (a), (b), (c) and (d), respectively.

values of the Reynolds number of the middle layer, with the values of the other parameters fixed, as given in the caption of Figure 4.4. In this figure the solid curve is plotted at the value $R e_{2}=100$, the value $R e_{2}=300$ corresponds to the dashed line, and the dotted curve represents the value $R e_{2}=1000$. Note that the regions above the wave number axis (i.e. the range to the left of the cutoff wave numbers) and below the curves are assumed to be unstable, according the positive sign of the real part of the complex frequency, while the areas below the wave number axis (i.e. the range right to the cutoff wave numbers) which represent the negative values of the disturbance growth rate, are stable. In this sense, we have three cutoff wave numbers which are $1.58,1.95$, and 2.1 corresponding to the values 100,300 , and 1000 of $R e_{2}$ respectively. Thus, it is obvious that as the Reynolds number increases both the growth rate and the cutoff wave numbers increase, and hence we conclude that the Reynolds number has a destabilizing influence on the movement of the layers (see [9]).

Figure 4.5 exhibits the effects of the the magnetic permeability ratio $\hat{\mu}_{e 1}(=$ $\left.\mu_{e 1} / \mu_{e 2}\right)$ on the stability behavior of the fluid layers. In this graph the solid, dashed and dotted curves represent the values $1.42,1.55$, and 1.72 of the ratio $\hat{\mu}_{e 1}$ respectively. Having noted the stability chart of this diagram, it is observed that the increasing of the magnetic permeability in the range $0<k<0.8$ leads to an extension in the width of the instability regions (the regions under the curves and above the wave number axis correspond to the positive sign of the disturbance growth rate). On the other hand the growth rates of instabilities with different $\hat{\mu}_{e 1}$ stay almost identical 

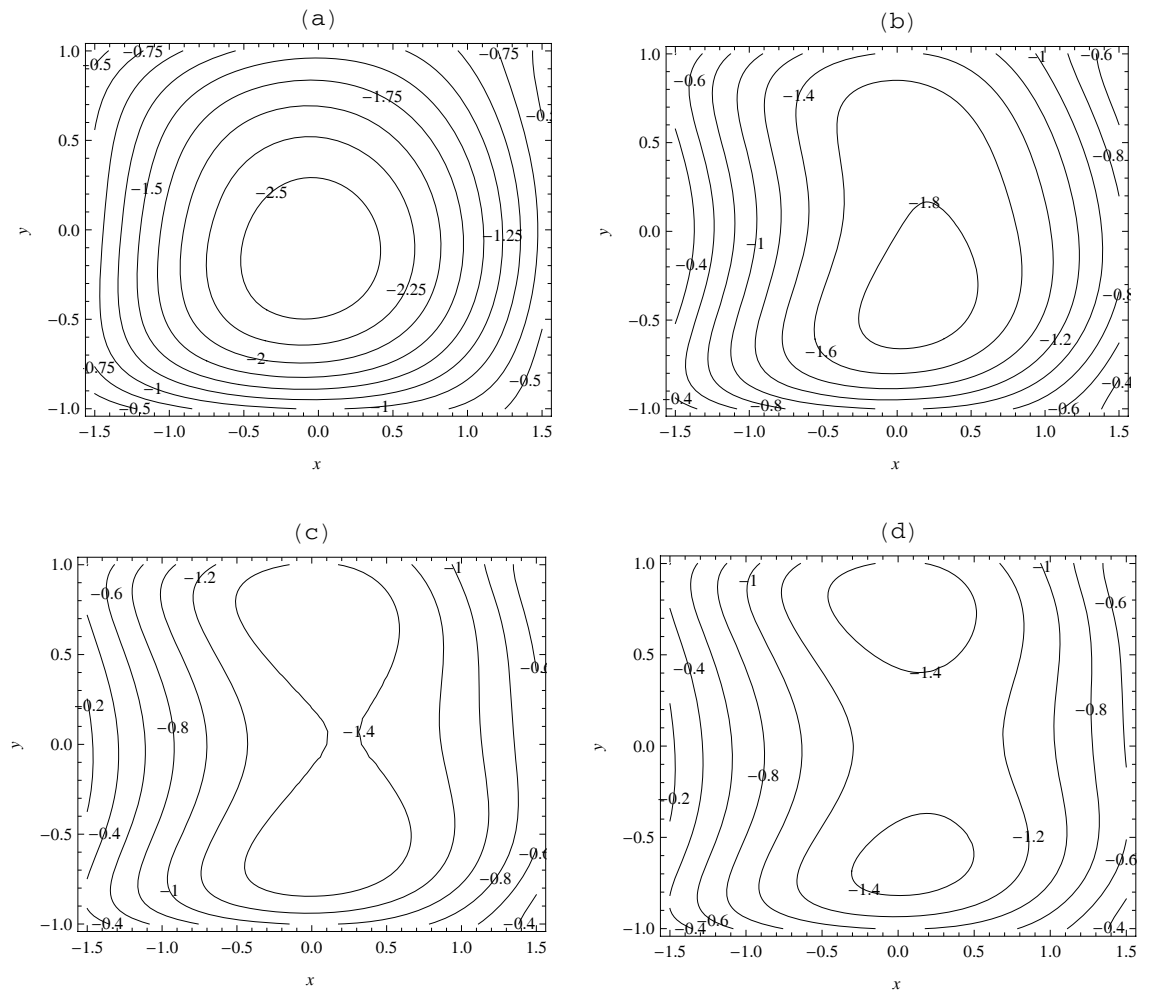

FIG. 4.10. The same system as that considered in Figure 4.9, while $R e_{2}=100,500,1000$ and 1300 of the parts (a), (b), (c) and (d), respectively.

for the wave numbers in the range $0.8<k<1.5$. In addition, the fluid sheet is stable in the region $0.8<k<0.25$ and both the cutoff wave numbers and the growth rates are increased due to the increasing of the magnetic permeability ratio $\hat{\mu}_{e 1}$. It is clear that the magnetic permeability $\hat{\mu}_{e 1}$ has a stabilizing effect on the movement of the fluid sheets. In other words, the horizontal field has a stabilizing influence on the movement of the waves when the magnetic permeability of the middle fluid $\mu_{e 2}$ is less than its counterpart of the lower fluid $\mu_{e 1}$.

The influence of changes of the magnetic permeability parameter $\hat{\mu}_{e 2}\left(=\mu_{e 3} / \mu_{e 2}\right)$, on the stability behavior in the plane $\left(\omega_{r}-k\right)$ is illustrated in Figure 4.6. The calculations are made for a system having the same parameters given in Figure 4.5, while the permeability parameter $\hat{\mu}_{e 2}$ has some variation for the sake of comparison. In the Figure 4.5 the values 1.983, 1.998, and 2.007 are selected for $\hat{\mu}_{e 2}$ correspond to the the solid, dashed, and dotted curves respectively. It is apparent from the inspection of Figure 4.6, under the influence of the permeability parameter $\hat{\mu}_{e 2}$, that the growth rates with different magnetic permeability ratios are almost identical for wave numbers less than 0.3 , but increase correspondingly at higher values of the wave number. Further, the plane $\left(\omega_{r}-k\right)$ is divided into two regions: the first is $0<k<1$, which represents a stabilizing effect for increasing the parameter $\hat{\mu}_{e 2}$, since in this range we notice that when the permeability ratio $\hat{\mu}_{e 2}$ is increased, both the growth rates and the cutoff wave numbers of non-Newtonian fluid sheets are decreased. The second region lies in the range $1<k<2$, and it is worthwhile to notice that the 

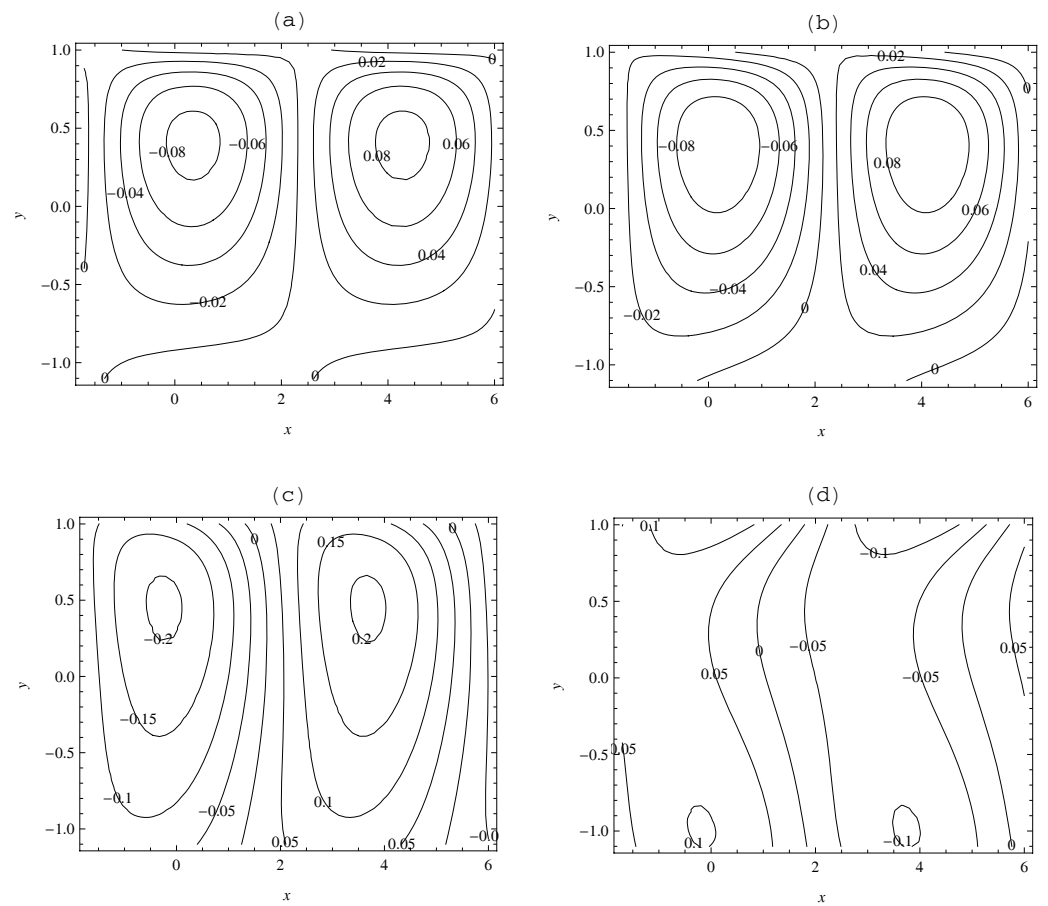

FIG. 4.11. The same system as that considered in Figure 4.10, while $Q_{1}=1,30,60$, and 65 on the partitions (a), (b), (c) and (d), respectively.

destabilizing role is found for the increasing the parameter $\hat{\mu}_{e 2}$, where a slight increase of the value of the magnetic permeability leads to a drastic increase in both the growth rates and the cutoff wave numbers. A general trend revealed by Figure 4.5 is that the phenomenon of the dual (irregular) role is observed for increasing the magnetic permeability $\hat{\mu}_{e 2}$ : one of the two roles is a stabilizing influence in the range $0<k<1$, and the other is a destabilizing influence in the range $1<k<2$.

An inspection of Figures 4.5 and 4.6 then shows that, broadly speaking, the ratio between the permeabilities $\mu_{e 1}, \mu_{e 3}$, and $\mu_{e 2}$ has a principle role in the administration of the behavior of the horizontal field. This suggests that when the permeability of the upper fluid $\mu_{e 3}$ is more than its counterpart of the middle fluid $\mu_{e 2}$, the field keeps its energy and absorbs a part of the kinetic energy of the surface waves. On the other hand, when the permeability of the inner fluid $\mu_{e 2}$ is less than that of the lower fluid $\mu_{e 1}$, the field sometimes keeps its energy and absorbs a parts of the kinetic energy of the interfacial waves, while transmitting its energy to these waves, which leads to instability of the motion of other waves.

In order to explore the effects of the retardation time $\lambda_{2}^{(2)}$ on the stability criteria, three values of $\lambda_{2}^{(2)}$ are collected in Figure 4.7 in the plane $\left(\omega_{r}-R e_{2}\right)$. In this figure we select the values $\lambda_{2}^{(2)}=0.4,0.5$, and 0.6 correspond to the solid, dashed, and dotted curves respectively. Investigation of the stability diagram of Figure 4.7 reveals that there are three values of the cutoff Reynolds number which are $R e_{2}=2.26,2.45,2.56$ correspond to the values $0.4,0.5$, and 0.6 of $\lambda_{2}^{(2)}$ respectively. Thus, we conclude that the increase of the cutoff $R e_{2}$ leads to an increase of the retardation time $\lambda_{2}^{(2)}$. On 
the other side the stability areas under the curves are contracted due to the increase of the retardation time $\lambda_{2}^{(2)}$. At this end, it can be demonstrated that the increase of the retardation time $\lambda_{2}^{(2)}$ plays a destabilizing role in the stability behavior. This result is in agreement with that obtained in [27].

Figure 4.8 illustrates the influence of the density ratio $\hat{\rho}_{1}\left(=\rho_{1} / \rho_{2}\right.$, the lower layer to the middle sheet) on the wave growth rate. The graph shown in the plane $\left(\omega_{r}-k\right)$ are achieved for three values of the ratio $\hat{\rho}_{1}=0.04,0.1$, and 0.16 , corresponding to the continuous, dashed, and dotted lines respectively, where the other quantities are held fixed. In this figure the areas lying under the $k$-axis and above the curves are stable and may be called stability regions (corresponding to the negative values of $\omega_{r}$ ). The inspection of the stability diagram of Figure 4.8 reveals that the increase of the density ratio $\hat{\rho}_{1}$ leads to increase in the width of the stability regions. The conclusion that may be drawn here is that the ratio $\hat{\rho}_{1}$ has a stabilizing influence on the stability behavior of the waves. This result confirms the fact that the system is stable when the lower fluid is more heavy than the upper.

4.3. Streamlines configuration. This section investigates the influence of the physical parameters (such as the porosity effect, Reynolds and Weber numbers) on the flow behavior in terms of the streamlines field (a curve formed by the velocity vectors of each fluid particle at a certain time is called a streamline, in which the tangent at each point of this curve indicates the direction of fluid at that point). The streamlines (curvilinear) in the physical domain are thus mapped into horizontal grid lines in the computational plane, resulting in a rectangular computational region. The streamlines are effective tools to visualize a qualitative impression of the flow behavior during the motion. The streamlines picture is achieved by fixing the value of all the physical parameters except for one. Snapshots of instantaneous streamlines of the stream function are shown in Figure 4.9-4.11.

The influence of the Weber number $W_{2}$ is presented throughout the parts of Figure 4.9 for a system having the same parameters considered in Figure 4.2, with $R_{2}=$ $500, k=0.8, t=0.1, \hat{\xi}_{1}=0.05, \hat{\xi}_{2}=0.07$. Inspection of Figure 4.9 (a) reveals that the flow consists of two cells (contours) consisting of clockwise (right, positive values of streamlines) and anti clockwise (left, negative values of streamlines) circulations. In parts (b) and (c) of this graph, the values of $W_{2}$ are increased to 5 and 8 respectively. It is worthwhile to notice that streamlines contours are divided into four cells, two of which have positive values (clockwise) which lie in the upper right and bottom left sides and the others are negative (anti clockwise). Further increase of $W_{2}(=10)$ is introduced in part (d) of the 4.9. It is discovered from this part that the contours again are transformed into two sets of cells and this is due to the increasing of the Weber number. A conclusion that may be made from the comparison among the parts (a-d) of Figure 4.9 is that higher Weber numbers increase the concentration of the streamlines in the movement of the fluids.

Figure 4.10 illustrates streamlines under the same values considered in the above system of Figure 4.9, while the Reynolds number $R e_{2}$ has four different values $(=100,500,1000,1300)$ for the sake of comparison. It is shown that the flow consists of cells (contours) consisting of anti clockwise circulations. It is observed from Figure 4.10 (a), (b) that the increasing of the Reynolds number leads to a reduction in the density of the streamlines cells. In part (c) of this graph, we note that the middle contour of the streamlines is contracted at the center, until it is divided into two contours in part (d) of Figure 4.10.

Figure 4.11 is made in order to see the effects of porosity in the lower layer of the 
fluid on the streamlines distribution, for the same parameters considered in Figure 4.9 and at four different values of the porous parameter $Q_{1}$ varying from 1 to 65 . In light of the stability configuration, we notice that corresponding to the parts (a-d) of Figure 4.11 there are four different values of the disturbance growth rate $\left(\omega_{r}\right)$, which are $-0.034,-0.135,-0.603$ and 0.396 . Since the stability of fluid sheets arises from negative growth rates, thus it can be observed that the parts (a-c) of Figure 4.11 represent streamlines for stable system while in Figure 4.11(d) we deduce that the central contour moves towards up and down and decreases in size when the system is unstable.

\section{Stability of two layers as a special case}

The main goal of this section is to investigate the limiting case when the thickness of the inner layer tends to zero (i.e. $a \rightarrow 0$ ). Define the characteristic dimensionless length as $\sqrt{T / g \rho_{2}}$ (since for any physical quantities defined above the medium 3 tends to the medium 2, for example $\rho_{3} \rightarrow \rho_{2}, \mu_{3} \rightarrow \mu_{2}$ ). At this stage the dispersion equation, which controls the stability process of the interface between the two superimposed non-Newtonian fluids, is

$$
\begin{aligned}
& \left\{\hat{\mu}_{1}(\omega)\left(2 i k^{2}-\frac{1}{Q_{1} R e_{2}}\right)-\hat{\rho}_{2} \omega\right\} \tilde{A}_{1}^{(1)}+\left\{\hat{\mu}_{2}(\omega)\left(2 i k^{2}-\frac{1}{Q_{2} R e_{2}}\right)+\hat{\rho}_{1} \omega\right\} \tilde{A}_{2}^{(2)} \\
& \quad+2 i k\left\{L_{1} \hat{\mu}_{1}(\omega) \tilde{A}_{3}^{(1)}+L_{2} \hat{\mu}_{2}(\omega) \tilde{A}_{4}^{(2)}\right\}+k H^{(1)}\left\{\left(\hat{\mu}_{e 1} \sin \left(\theta_{1}\right)-i \cos \left(\theta_{1}\right)\right) \tilde{B}_{1}^{(1)}\right. \\
& \left.\quad+\hat{\mu}_{e 1}\left(\sin \left(\theta_{1}\right)-i \cos \left(\theta_{1}\right)\right) \tilde{B}_{2}^{(2)}\right\}+\hat{\rho}_{1}+k^{2} W-1=0
\end{aligned}
$$

where,

$$
\begin{aligned}
\tilde{A}_{1}^{(1)} & =\frac{i \omega\left[\tilde{A}_{0}\left(k-L_{1}\right)-2 k\left(k \hat{\mu}_{1}(\omega)+L_{2} \hat{\mu}_{2}(\omega)\right)\right]}{k\left(k-L_{1}\right) \tilde{A}_{0}}, \\
\tilde{A}_{3}^{(1)} & =\frac{2 i \omega\left(k \hat{\mu}_{1}(\omega)+L_{2} \hat{\mu}_{2}(\omega)\right)}{\left(k-L_{1}\right) \tilde{A}_{0}} \\
\tilde{A}_{0} & =\hat{\mu}_{1}(\omega)\left(k+L_{1}\right)+\hat{\mu}_{2}(\omega)\left(k+L_{2}\right), \\
\tilde{A}_{2}^{(2)} & =\frac{i \omega\left[\tilde{A}_{0}\left(k-L_{2}\right)-2 k\left(L_{1} \hat{\mu}_{1}(\omega)+k \hat{\mu}_{2}(\omega)\right)\right]}{k\left(k-L_{2}\right) \tilde{A}_{0}}, \\
\tilde{A}_{4}^{(2)} & =\frac{2 i \omega\left(L_{1} \hat{\mu}_{1}(\omega)+k \hat{\mu}_{2}(\omega)\right)}{\left(k-L_{2}\right) \tilde{A}_{0}}, \quad m=1,2 . \\
\tilde{B}_{m}^{(m)} & =\frac{(-1)^{m-1} H^{(1)}\left(1-\hat{\mu}_{e 1}\right)\left[\hat{\mu}_{e 1}^{m-1} \sin \left(\theta_{1}\right)+i \cos \left(\theta_{1}\right)\right]}{\left(1+\hat{\mu}_{e 1}\right)}, \quad m
\end{aligned}
$$

Equation (5.1) is the general equation describing two-dimensional motion of one interface between two magnetic non-Newtonian fluids. It accounts for gravity, porosity effects, and viscoelastic and magnetic stresses. A special case can be obtained from Equation (5.1), which is the case of a clear fluid (non-porous medium, i.e. $Q_{j} \rightarrow \infty$ or $\phi_{j}=0$ ) in which highly viscous fluids are considered. Under these assumptions, we 


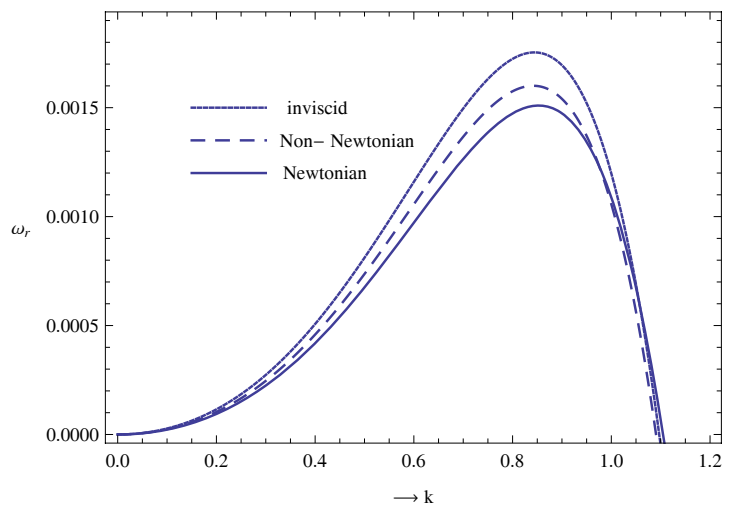

FIG. 5.1. Non-dimensional growth rate of different fluids versus non-dimensional wave number at $H^{(1)}=1, \theta_{1}=0, \hat{\mu}_{e 1}=2, \hat{\rho}_{1}=2, \quad \hat{\mu}_{1}=0.2, R e_{2}=500, Q_{1}=8, Q_{2}=1, W=1, \lambda_{1}^{(1)}=0.5, \lambda_{2}^{(1)}=$ $0.4, \lambda_{1}^{(2)}=0.5$ and $\lambda_{2}^{(2)}=0.8$.

have

$$
L_{l}=\left[k^{2}+\frac{R e_{2} \hat{\rho}_{l} \omega}{\hat{\mu}_{l}}\right]^{\frac{1}{2}}=k\left[1+\frac{R e_{2} \hat{\rho}_{l} \omega}{2 k^{2} \hat{\mu}_{l}}\right], \quad l=1,2,
$$

so that

$$
L_{l}-k=\frac{R e_{2} \hat{\rho}_{l} \omega}{2 k^{2} \hat{\mu}_{l}}
$$

Substituting the values of $L_{1}$ and $L_{2}$ from Equation (5.3) into Equation (5.1) and simplifying, we obtain the dispersion relation

$$
\omega^{5}+\left(\hat{\alpha}_{11}+i \hat{\alpha}_{12}\right) \omega^{4}+\left(\hat{\alpha}_{21}+i \hat{\alpha}_{22}\right) \omega^{3}+\left(\hat{\alpha}_{31}+i \hat{\alpha}_{32}\right) \omega^{2}+\left(\hat{\alpha}_{41}+i \hat{\alpha}_{42}\right) \omega+\hat{\alpha}_{51}+i \hat{\alpha}_{52}=0,
$$

where the coefficient $\hat{\alpha}$ 's are clear from the context. Equations similar to (5.4) have been obtained by Kumar and Singh [19] and Sunil et al [28].

5.1. Applications. In Figures 5.1-5.7, our aim is to determine the numerical profiles for the stability pictures of the waves propagating through the interface which separates two superposed non-Newtonian fluid layers in a porous medium. In order to facilitate this examination, numerical calculations for the dispersion Equation (5.1) are performed. Figure 5.1 shows the variation of the non-dimensional growth rate disturbances versus the non-dimensional wave number for different fluid layers for a system having $H^{(1)}=1, \theta_{1}=0, \hat{\mu}_{e 1}=2, \hat{\rho}_{1}=2, \hat{\mu}_{1}=0.2, R e_{2}=500, Q_{1}=8, Q_{2}=$ $1, W=1, \lambda_{1}^{(1)}=0.5, \lambda_{2}^{(1)}=0.4, \lambda_{1}^{(2)}=0.5$, and $\lambda_{2}^{(2)}=0.8$. It is obvious that in Figure 5.1 the maximum growth rate for any non-Newtonian fluid is larger than that of a Newtonian fluid sheet and smaller than that of an inviscid fluid sheet. Therefore, we conclude that in the range of flow parameters associated with the results of Figure 5.1, a sheet of such a non-Newtonian fluid is more unstable than a sheet of Newtonian liquid against small disturbances. This behavior coincides with that observed in Figure 4.1 in the case of two interfaces between three layers. 


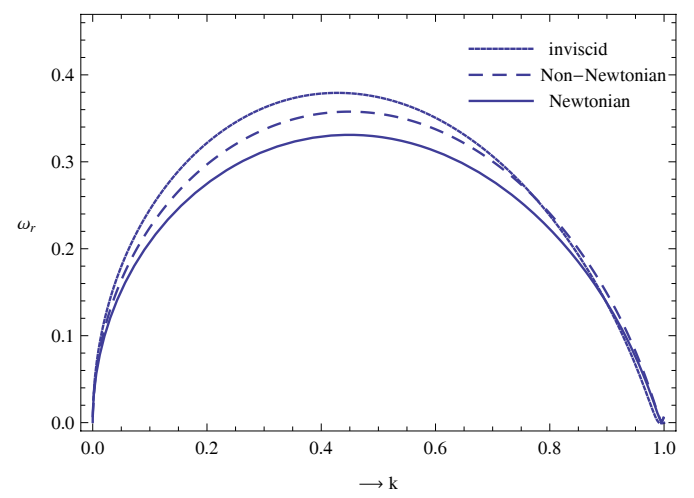

FIG. 5.2. Refers to the same system considered in Figure 5.1 in the limiting case of non porous medium.

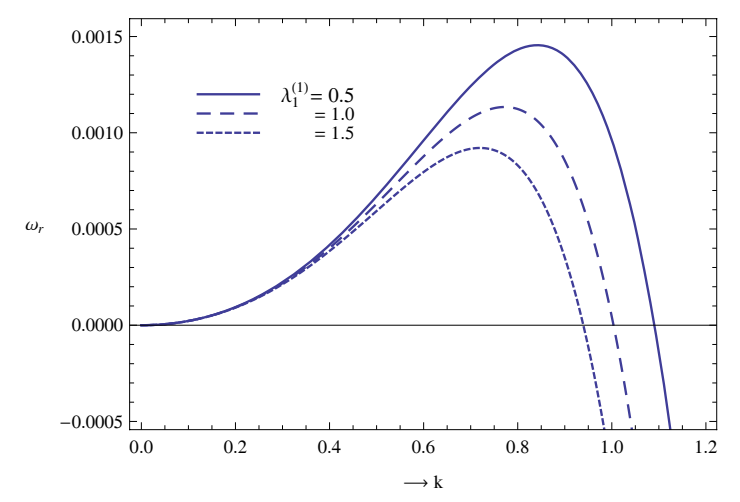

FIG. 5.3. Refers to the same system considered in Figure 5.2, but with $\lambda_{1}^{(1)}=0.5$ (solid), 1 (dashed), 1.5 (dotted).

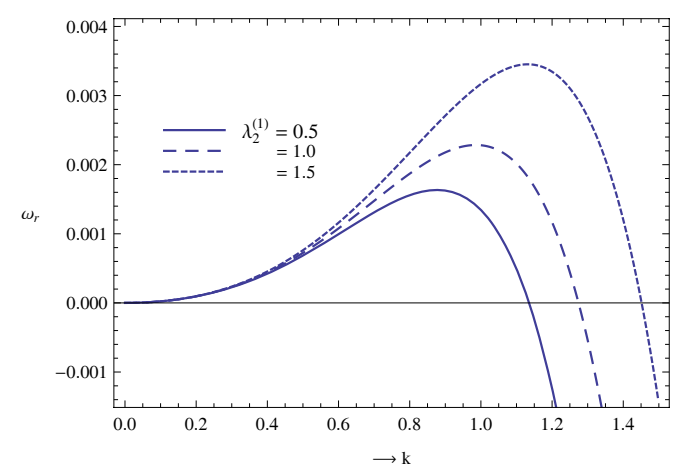

FIG. 5.4. Considered the same system given in Figure 5.2, but with $\lambda_{2}^{(1)}=0.5,1,1.5$. 


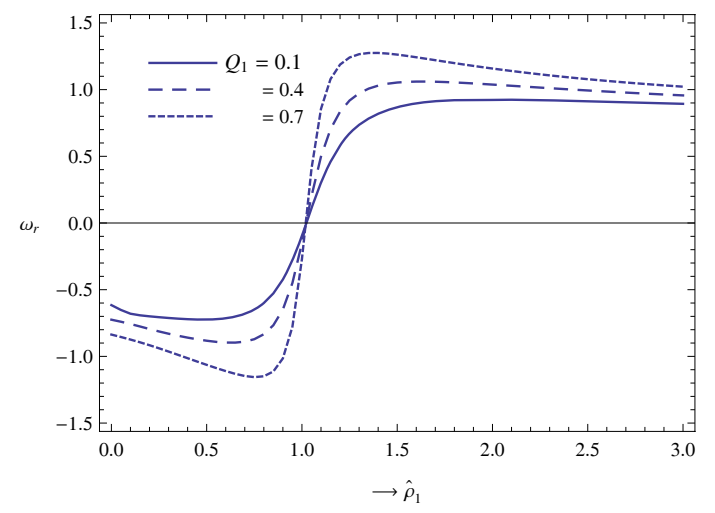

FIG. 5.5. Represents the stability diagrams in the plane $\left(\omega_{r}-\hat{\rho}_{1}\right)$ at $H^{(1)}=0.5, \theta_{1}=0, k=$ $1, \hat{\mu}_{e 1}=1.2, \hat{\mu}_{1}=0.1, R e_{2}=1000, Q_{2}=2, W=5, \lambda_{1}^{(1)}=0.1, \lambda_{2}^{(1)}=0.4, \lambda_{1}^{(2)}=0.5$, and $\lambda_{2}^{(2)}=0.15$, with $Q_{1}=0.2,0.7$, and 1.1 .

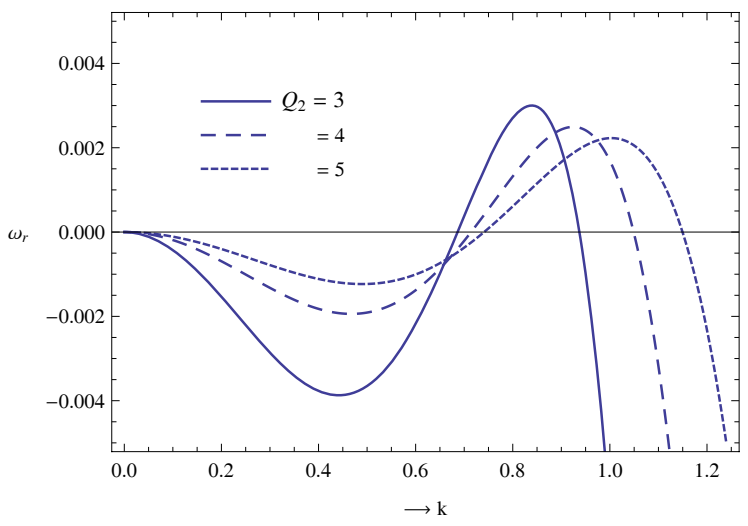

FIG. 5.6. Shows the effects of the porous parameter $Q_{2}$ on the growth rates in the plane $\left(\omega_{r}-\right.$ $k$ ), for a system having $H^{(1)}=0.1, \theta_{1}=0, \hat{\rho}_{1}=0.5, \hat{\mu}_{e 1}=2, \hat{\mu}_{1}=2, R_{2}=800, Q_{1}=0.8, W=$ $1.5, \lambda_{1}^{(1)}=0.01, \lambda_{2}^{(1)}=0.04, \lambda_{1}^{(2)}=0.05$, and $\lambda_{2}^{(2)}=0.08$, with $Q_{2}=2.3$, 2.6, and 3.2.

In the limiting case of non porous media (i.e. continuum fluids), numerical calculations for Equation (5.1) are made. The results for calculations are displayed in Figure 5.2, in the plane $\left(\omega_{r}-k\right)$. The analysis displayed in this figure further confirms that the non-Newtonian fluid sheets are more unstable than Newtonian ones.

In summary of Figure 4.1, 4.2 (two interfaces) and Figure 5.1, 5.2 (one interface), for non-Newtonian fluids, both viscosity and elasticity effects may exist at the same time, which makes the instability behavior much more complicated than for Newtonian fluids. In general, the fluid viscosity tends to weaken the instability in comparison with inviscid fluids through decreasing the disturbance growth rate, whereas fluid elasticity results in an enhancement of the instability. Under the common action of the fluid viscosity and elasticity effects, the growth rate curve of non-Newtonian fluid sheets should lie between those of inviscid and Newtonian ones. On the basis of the above analysis, it is believed that the area between the growth rate curves of the 


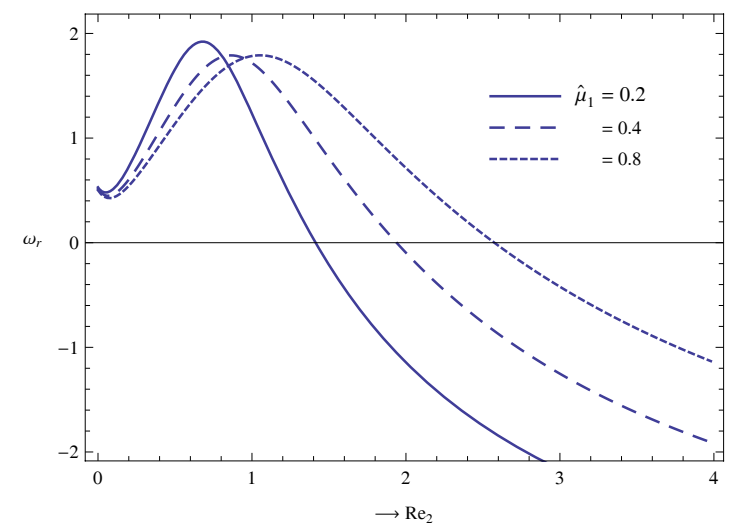

Fig. 5.7. Illustrated in the plane $\left(\omega_{r}-R e_{2}\right)$ at $H^{(1)}=10, \theta_{1}=90, \hat{\rho}_{1}=0.5, \hat{\mu}_{e 1}=2, \quad k=$ $1, Q_{1}=7, Q_{2}=8, W=4, \lambda_{1}^{(1)}=0.01, \lambda_{2}^{(1)}=0.04, \lambda_{1}^{(2)}=0.05$, and $\lambda_{2}^{(2)}=0.1$, with viscosity ratio $\hat{\mu}_{1}=0.2,0.4,0.8$.

inviscid and viscoelastic fluid sheets is induced by the interaction of the fluid viscosity and elasticity effects in fluids. In this sense, this area is called the viscoelasticityinduced region, and the area between the growth rate curves of the Newtonian and non-Newtonian fluid sheets is termed the elasticity enhanced region.

The effects of both relaxation time $\lambda_{1}^{(1)}$ and the retardation time $\lambda_{2}^{(1)}$ on the evolution of stabilities of non-Newtonian fluid sheets are presented in Figure 5.3 and Figure 5.4 in the plane $\left(\omega_{r}-k\right)$. In these two graphs the system is selected for the the same values considered in Figure 5.1, where in Figure 5.3 the relaxation time $\lambda_{1}^{(1)}$ is varied stepwise from 0.5 to 1.5. The inspection of Figure 5.3 indicates that as the relaxation time is increased both the growth rates and the cutoff wave numbers are reduced, or alternatively that the unstable regions under the curves are decreased. Therefore it is concluded that the relaxation time effects have a stabilizing influence in the fluid sheets. In Figure 5.4, the retardation time $\lambda_{2}^{(1)}$ is also varied stepwise from 0.5 to 1.5 with $\lambda_{1}^{(1)}=0.4$. Having checked the stability picture of this figure, it is discovered that increasing the retardation time leads to growth of the instability areas above the wave number axis, and consequently the retardation time has a destabilizing effect. On the other hand from the comparison of Figure 5.3 and Figure 5.4 we deduce that increasing the value $\lambda_{2}^{(1)} / \lambda_{1}^{(1)}$, which is the ratio of deformation retardation time to stress relaxation time (the time constant ratio for short), has a destabilizing influence on the considered system. Goldin et al [29] have obtained a similar conclusion in their studies of axisymmetric instability of non-Newtonian jets.

Figures 5.5 and 5.6 display the effects of the porosity through the porous parameters on the growth rate of non-Newtonian fluid sheets. In Figure 5.5 the influence of the porous parameter $Q_{1}$ on the stability of the fluid layers is depicted in a system with parameters $H^{(1)}=0.5, \theta_{1}=0, k=1, \hat{\mu}_{e 1}=1.2, \hat{\mu}_{1}=0.1, \quad R e_{2}=1000, Q_{2}=$ $2, W=5, \lambda_{1}^{(1)}=0.1, \lambda_{2}^{(1)}=0.4, \lambda_{1}^{(2)}=0.5$, and $\lambda_{2}^{(2)}=0.15$, with $Q_{1}=0.1,0.4$, and 0.7 in solid, dashed and dotted curves respectively, and in which the growth rates are plotted against the density ratio $\hat{\rho}_{1}$. It is seen from Figure 5.5 that the system is stable in the range $\hat{\rho}_{1}<1$ (corresponding to the negative values of the growth rates) and unstable in the range $1<\hat{\rho}_{1}<3$ (corresponding to the positive values of $\omega_{r}$ ). In addition, 
increasing the porous parameter $Q_{1}$ in the range $\hat{\rho}_{1}<1$ leads to decreasing growth rates, which means that the porous parameter $Q_{1}$ plays a stabilizing role. Moreover, when the porous parameter $Q_{1}$ increases in the region $1<\hat{\rho}_{1}<3$, the instability areas under the curves increases, i.e. $Q_{1}$ has a destabilizing effect on the movement of the fluid layers. In general, from Figure 5.5, it is noticed that there are two roles played by the variation of the the porous parameter $Q_{1}$ : the first one is stabilizing when the density ratio $\hat{\rho}_{1}$ less than the value 1 , and the other role is destabilizing when $\hat{\rho}_{1}$ lies between the values 1 and 2 . Hence the phenomenon of the dual role is found for increasing the porous parameter $Q_{1}$.

Figure 5.6 shows the effects of the porous parameter $Q_{2}$ on the growth rates in the plane $\left(\omega_{r}-k\right)$, where the values $2.3,2.6$, and 3.2 are chosen for $Q_{2}$. It is obvious from this graph that for every value of the $Q_{2}$ the corresponding curve crosses the wave number axis at three points (the cutoff wave numbers) and forms three stability and instability regions. Two of these regions lie under the $k$-axis and are stable (negative $\omega_{r}$-axis), while the area which lies above the $k$-axis and under every curve is unstable (positive $\omega_{r}$ - axis). Inspection of Figure 5.7 revels that increasing the porous parameter $Q_{2}$ in the range $k<0.7$ induces a contraction in the width of the stability regions (stabilizing role), whereas the instability region is reduced by increasing $Q_{2}$ in the range $0.7<k<1.2$ (destabilizing effect). It is clear that from Figure 5.5 and Figure 5.6 that both of the porous parameters $Q_{1}$ and $Q_{2}$ have a dual influence on the stability of the movement of the waves.

The change of the lower to the upper fluid viscosity ratio $\hat{\mu}_{1}$ in the stability criteria is illustrated in Figure 5.7. The graph shown in the plane $\left(\omega_{r}-R e_{2}\right)$ is calculated for three values of the viscosity ratio $\hat{\mu}_{1}=0.2,0.4$, and 0.8 , where the other quantities are held fixed as considered in Figure 5.6 with $Q_{2}=0.7$. It is observed that from Figure 5.7 there are three cutoff (critical) Reynolds numbers corresponding to the three values of the above viscosity ratio $\left(R e_{2}=1.4,1.9\right.$ and 2.6$)$, indicating that increasing the viscosity ratio results in an increasing growth rate of disturbances on the fluid sheets, which destabilizes the fluid sheets. A similar result was reported by Ozen et al [30] in their studies of electrohydrodynamic linear stability of two immiscible fluids in channel flow.

\section{Conclusions}

The stability properties of two-dimensional non-Newtonian fluid layers moving in porous media under the influence of uniform magnetic field were investigated. The solution of the linearized equations of motion under the boundary conditions leads to an implicit dispersion relation between the growth rate and wave number. The limiting case of the stability of one interface between two fluids has been discussed. The stability analysis has been performed theoretically and numerically with the physical parameters are put in the dimensionless form. Some stability diagrams have been plotted and discussed. The stability examination yields the following results:

1. Non-Newtonian fluid sheets have a higher growth rate than Newtonian fluid sheets for both three layer- and two layer- types of disturbances, indicating that non-Newtonian fluid sheets are more unstable than Newtonian fluid sheets. This result is in agreement with that obtained by Liu et al [9].

2. For the fluid sheets, the viscoelasticity-induced region in the plots of growth rate versus wave number results from the interaction of the fluid viscosity and the elasticity effects; the fluid elasticity tends to damp the stability, whereas the fluid viscosity enhances stability. 
3. It has been found that increasing the viscosity coefficient ratio as well as the porosity damps the growth rate, where a part of its kinetic energy may be absorbed. However, it is expected that a more careful investigation would show that the motion of the interfacial waves will be more stable after increasing the value of the viscosity as well as the porosity.

4. Increasing the ratio of deformation retardation time to stress relaxation time $\lambda_{2}^{(1)} / \lambda_{1}^{(1)}$ (the time constant ratio) has a destabilizing influence on the stability criteria. A similar result was reported in [27]. Also, Goldin et al [29] have obtained a similar conclusion in their studies of axisymmetric instability of non-Newtonian jets.

5. The horizontal magnetic field plays a stabilizing role, and the vertical plays a destabilizing role on the stability behavior in the cases of two interfaces and one interface among the fluids. In other words, when the magnetic field is increased, the field sometimes keeps its energy and absorbs a part of the kinetic energy of the interfacial waves while transmitting its energy to these waves, which leads to the instability in the motion of the waves.

6. A destabilizing effect is observed by increasing of Reynolds number. On the other hand, the phenomenon of the dual role is found for increasing the magnetic permeability ratio on the movement on the fluid. A similar result was reported by Ozen et al [30] in their studies of electrohydrodynamic linear stability of two immiscible fluids in channel flow.

7. An increase of the lower-to-upper fluid density ratio enhances both the growth rate and the instability range of the fluid sheet, whereas the converse is true for increasing the upper-to-lower fluid density ratio, leading to an enhancement of stability.

Appendix A. The values of the coefficients appearing in Equation (3.12) are

$$
\begin{aligned}
A_{11}^{(1)}= & \frac{-2 i \omega \hat{\mu}_{2}(\omega) e^{k+2\left(L_{2}+k\right)}}{k\left(k-L_{1}\right) e^{2\left(L_{2}+k\right)} A_{0}}\left\{\hat { \mu } _ { 1 } ( \omega ) ( k ^ { 2 } + L _ { 1 } ^ { 2 } ) \left[\hat { \mu } _ { 2 } ( \omega ) ( k ^ { 2 } - L _ { 2 } ^ { 2 } ) \left(k \cosh (2 k) \sinh \left(2 L_{2}\right)-L_{2} \cosh \left(2 L_{2}\right)\right.\right.\right. \\
& \left.\times \sinh (2 k))+\hat{\mu}_{3}(\omega)\left(k+L_{3}\right)\left(2 k\left(L_{2}-L_{2} \cosh \left(2 L_{2}\right) \cosh (2 k)\right)+\left(k^{2}+L_{2}^{2}\right) \sinh (2 k) \sinh \left(2 L_{2}\right)\right)\right] \\
& +\hat{\mu}_{2}(\omega)\left[\hat { \mu } _ { 2 } ( \omega ) ( k ^ { 2 } - L _ { 2 } ^ { 2 } ) \left(2 k^{2} L_{2} \cosh \left(2 L_{2}\right) \sinh (2 k)+\sinh \left(2 L_{2}\right)\left(L_{1}\left(k^{2}-L_{2}^{2}\right) \sinh (2 k)-k\left(k^{2}\right.\right.\right.\right. \\
& \left.\left.\left.\left.+L_{2}^{2}\right) \cosh (2 k)\right)\right)\right]+\hat{\mu}_{3}(\omega)\left(k+L_{3}\right)\left[L_{2} \cosh \left(2 L_{2}\right)\left(k\left(3 k^{2}+L_{2}^{2}\right)-L_{1}\left(k^{2}-L_{2}^{2}\right) \sinh (2 k)\right)-k\right. \\
& \left.\left.\times \sinh \left(2 L_{2}\right)\left(k\left(k^{2}+3 L_{2}^{2}\right) \sinh (2 k)-L_{1}\left(k^{2}-L_{2}^{2}\right) \cosh (2 k)\right)-k\left(3 k^{2} L_{2}+L_{2}^{3}\right)\right]\right\}, \\
A_{0}= & 2 \hat{\mu}_{2}(\omega)\left(k^{2}-L_{2}^{2}\right)\left[\hat{\mu}_{2}(\omega)\left(k^{2}-L_{2}^{2}\right) \sinh (2 k) \sinh \left(2 L_{2}\right)+\hat{\mu}_{2}(\omega)\left(k+L_{3}\right)\left(k \cosh (2 k) \sinh \left(2 L_{2}\right)\right.\right. \\
& \left.\left.-L_{2} \cosh \left(2 L_{2}\right) \sinh (2 k)\right)\right]+2 \hat{\mu}_{1}(\omega)\left(k+L_{2}\right)\left[\hat { \mu } _ { 2 } ( \omega ) ( k ^ { 2 } - L _ { 2 } ^ { 2 } ) \left(k \cosh (2 k) \sinh \left(2 L_{2}\right)-L_{2}\right.\right. \\
& \left.\times \cosh \left(2 L_{2}\right) \sinh (2 k)\right)+\hat{\mu}_{3}(\omega)\left(k+L_{3}\right)\left(2 k L_{2}\left(1-\cosh (2 k) \cosh \left(2 L_{2}\right)\right)+\left(k^{2}+L_{2}^{2}\right) \sinh (2 k)\right. \\
& \left.\left.\times \sinh \left(2 L_{2}\right)\right)\right], \\
& \\
& \left.\quad \times\left(k\left(k-L_{3}\right)\left(1-e^{4 L_{2}}\right) e^{2 k}+L_{2}\left(\left(k+L_{3}\right)\left(1+-e^{4 L_{2}}\right) e^{2 k}-2 e^{2 L_{2}}\left(k-L_{3} e^{4 k}\right)\right)\right)\right\}, \\
A_{12}^{(1)}= & \frac{-i \omega\left(k^{2}-L_{2}^{2}\right) e^{k}}{\left(k-L_{1}\right) e^{2\left(L_{2}+k\right)} A_{0}}\left\{2 \hat{\mu}_{2}(\omega)\left(\left(k^{2}+L_{2}^{2}\right) \sinh \left(2 L_{2}\right)-2 k L_{2} \sinh 2 k\right) e^{2\left(k+L_{2}\right)}+\hat{\mu}_{3}(\omega)\right. \\
& \quad(k)
\end{aligned}
$$




$$
\begin{aligned}
& A_{31}^{(1)}=\frac{i \omega e^{L_{1}}}{\left(k-L_{1}\right) e^{2\left(L_{2}+k\right)} A_{0}}\left\{k \hat { \mu } _ { 1 } ( \omega ) \left[4 \hat{\mu}_{2}(\omega)\left(k^{2}-L_{2}^{2}\right)\left(k \cosh (2 k) \sinh \left(2 L_{2}\right)-L_{2} \cosh \left(2 L_{2}\right) \sinh (2 k)\right)\right.\right. \\
& \times e^{2\left(k+L_{2}\right)}+\hat{\mu}_{3}(\omega)\left(k+L_{3}\right)\left(k^{2}\left(1-e^{4 k}\right)\left(1-e^{4 L_{2}}\right)+4 L_{2}\left(2 k\left(1-\cosh (2 k) \cosh \left(2 L_{2}\right)\right)+L_{2}\right.\right. \\
& \left.\left.\left.\times \sinh (2 k) \sinh \left(2 L_{2}\right)\right) e^{2\left(k+L_{2}\right)}\right)\right]+\hat{\mu}_{2}(\omega)\left[\hat { \mu } _ { 2 } ( \omega ) ( k ^ { 2 } - L _ { 2 } ^ { 2 } ) \left(k^{2}\left(1-e^{4 L_{2}}\right)-L_{2}\left(k\left(1+e^{4 L_{2}}\right)\right.\right.\right. \\
& \left.\left.-e^{4 k}\left(k+L_{2}+\left(k-L_{2}\right) e^{4 L_{2}}\right)\right)\right)-\hat{\mu}_{3}(\omega)\left(k+L_{3}\right)\left(k^{3}\left(1-e^{4 L_{2}}\right)+\left(2\left(3 k^{2}+L_{2}^{2}\right) e^{2\left(k+L_{2}\right)}-e^{4 k}\right.\right. \\
& \left.\left.\left.\left.\times\left(e^{4 L_{2}}\left(k-L_{2}\right)^{2}+\left(k+L_{2}\right)^{2}\right)-k\left(2 k-L_{2}+e^{4 L_{2}}\left(2 k+L_{2}\right)\right)\right)\right)\right]\right\}, \\
& A_{32}^{(1)}=\frac{i \omega \hat{\mu}_{2}(\omega)\left(k^{2}-L_{2}^{2}\right) e^{L_{1}}}{\left(k-L_{1}\right) e^{2\left(L_{2}+k\right)} A_{0}}\left\{2 \hat{\mu}_{2}(\omega) e^{2\left(k+L_{2}\right)}\left(\left(k^{2}+L_{2}^{2}\right) \sinh \left(2 L_{2}\right)-2 k L_{2} \sinh (2 k)\right)+\hat{\mu}_{3}(\omega)\right. \\
& \left.\times\left(k\left(k-L_{3}\right)\left(1-e^{4 L_{2}}\right) e^{2 k}-L_{2}\left(2 k e^{2 L_{2}}+2 L_{3} e^{2\left(2 k+L_{2}\right)}-\left(k+L_{3}\right)\left(1+e^{4 L_{2}}\right) e^{2 k}\right)\right)\right\}, \\
& A_{11}^{(2)}=\frac{-i \omega e^{2 L_{2}+k}}{k e^{2\left(L_{2}+k\right)} A_{0}}\left\{\hat { \mu } _ { 2 } ( \omega ) \left[\hat{\mu}_{2}(\omega)\left(L_{2}^{4}-k^{4}\right) \sinh \left(2 L_{2}\right)+\hat{\mu}_{3}(\omega)\left(L_{3}+k\right)\left(( L _ { 2 } ^ { 2 } + k ^ { 2 } ) \left(k \sinh \left(2 L_{2}\right)\right.\right.\right.\right. \\
& \left.\left.\left.+L_{2} \cosh \left(2 L_{2}\right)\right)-2 L_{2} k^{2} e^{2 k}\right)\right]+\hat{\mu}_{1}(\omega)\left[\hat { \mu } _ { 2 } ( \omega ) ( k ^ { 2 } - L _ { 2 } ^ { 2 } ) \left(k\left(k-L_{1}\right) \sinh \left(2 L_{2}\right)-L_{2}\right.\right. \\
& \left.\times\left(k+L_{1}\right) \cosh \left(2 L_{2}\right)\right)+\hat{\mu}_{3}(\omega)\left(k+L_{1}\right)\left(2 k L_{1} L_{2} \cosh \left(2 L_{2}\right)-2 k L_{1} L_{2} e^{2 k}+\sinh \left(2 L_{2}\right)\right. \\
& \left.\left.\left.\times\left(k^{2}\left(L_{1}-k\right)+L_{2}^{2}\left(L_{1}+k\right)\right)\right)\right]\right\}, \\
& A_{12}^{(2)}=\frac{-i \omega e^{2 L_{2}+k}}{k e^{2\left(L_{2}+k\right)} A_{0}}\left\{\hat { \mu } _ { 2 } ( \omega ) ( k ^ { 2 } - L _ { 2 } ^ { 2 } ) e ^ { 2 k } \left[\hat{\mu}_{3}(\omega) L_{2}\left(k+L_{3}\right) \cosh \left(2 L_{2}\right)+\sinh \left(2 L_{2}\right)\left(\hat{\mu}_{2}(\omega)\left(k^{2}+L_{2}^{2}\right)\right.\right.\right. \\
& \left.\left.-k \hat{\mu}_{3}(\omega)\left(k-L_{3}\right)\right)\right]+\hat{\mu}_{1}(\omega)\left(k+L_{1}\right)\left[2 k^{2} L_{2}\left(\hat{\mu}_{2}(\omega)-\hat{\mu}_{3}(\omega)\right)-L_{2} \cosh \left(2 L_{2}\right)\left(\hat { \mu } _ { 2 } ( \omega ) \left(k^{2}\right.\right.\right. \\
& \left.\left.\left.\left.+L_{2}^{2}\right)-2 k^{2} \hat{\mu}_{3}(\omega)\right) e^{2 k}+\sinh \left(2 L_{2}\right)\left(k \hat{\mu}_{2}(\omega)\left(k^{2}+L_{2}^{2}\right)-\hat{\mu}_{3}(\omega)\left(k^{2}\left(k-L_{3}\right)+L_{2}^{2}\left(k+L_{3}\right)\right)\right) e^{2 k}\right]\right\}, \\
& A_{21}^{(2)}=\frac{-i \omega e^{k}}{2 k e^{2\left(L_{2}+k\right)} A_{0}}\left\{\hat { \mu } _ { 2 } ( \omega ) \left[\left(k^{4}-L_{2}^{4}\right)\left(1-e^{4 L_{2}}\right) e^{2 k}+\hat{\mu}_{3}(\omega)\left(k+L_{3}\right)\left(\left(k+L_{2}\right)\left(k^{2}+L_{2}^{2}\right) e^{2 k}\right.\right.\right. \\
& \left.\left.-\left(k-L_{2}\right)\left(k^{2}+L_{2}^{2}\right) e^{2 k+4 L_{2}}-4 k^{2} L_{2} e^{2 L_{2}}\right)\right]+2 \hat{\mu}_{1}(\omega) e^{2 L_{2}}\left[\hat { \mu } _ { 2 } ( \omega ) ( k ^ { 2 } - L _ { 2 } ^ { 2 } ) \left(k\left(k-L_{1}\right)\right.\right. \\
& \left.\times \sinh \left(2 L_{2}\right)-L_{2}\left(k+L_{1}\right) \cosh \left(2 L_{2}\right)\right) e^{2 k}+\hat{\mu}_{3}(\omega)\left(k-L_{3}\right)\left(2 k^{2} L_{2}-2 k^{2} L_{2} \cosh \left(2 L_{2}\right)\right. \\
& \left.\left.\left.\times e^{2 k}+\sinh \left(2 L_{2}\right)\left(k^{2}\left(k-L_{1}\right)+L_{2}^{2}\left(k+L_{1}\right)\right) e^{2 k}\right)\right]\right\}, \\
& A_{22}^{(2)}=\frac{i \omega e^{2 L_{2}+k}}{k e^{2\left(L_{2}+k\right)} A_{0}}\left\{\hat { \mu } _ { 1 } ( \omega ) ( k + L _ { 1 } ) \left[\hat{\mu}_{2}(\omega)\left(2 k^{2} L_{2} e^{2 k}-\left(k^{2}+L_{2}^{2}\right)\left(k \sinh \left(2 L_{2}\right)+L_{2} \cosh \left(2 L_{2}\right)\right)\right)\right.\right. \\
& \left.-\hat{\mu}_{3}(\omega)\left(2 k L_{2} L_{3}\left(\cosh \left(2 L_{2}\right)-e^{2 k}\right)-k^{2}\left(k-L_{3}\right) \sinh \left(2 L_{2}\right)+L_{2}^{2}\left(k+L_{3}\right) \sinh \left(2 L_{2}\right)\right)\right]+\hat{\mu}_{2}(\omega) \\
& \left.\times\left(k^{2}-L_{2}\right)\left[\hat{\mu}_{3}(\omega) L_{2}\left(k+L_{3}\right) \cosh \left(2 L_{2}\right)+\left(\hat{\mu}_{2}(\omega)\left(k^{2}+L_{2}^{2}\right)-k \hat{\mu}_{3}(\omega)\left(k-L_{3}\right)\right) \sinh \left(2 L_{2}\right)\right]\right\}, \\
& A_{31}^{(2)}=\frac{i \omega e^{L_{2}}}{e^{2\left(L_{2}+k\right)} A_{0}}\left\{\hat{\mu}_{1}(\omega)\left(k+L_{3}\right)\left(L_{1}\left(k-L_{2}\right)\left(e^{2 L_{2}}-e^{2 k}\right)+k\left(k-L_{2}\right)\left(1-e^{2\left(k+L_{2}\right)}\right)\right)-\hat{\mu}_{2}(\omega)\right. \\
& \left.\times\left(k^{2}-L_{2}^{2}\right)\left(k+L_{2} e^{4 k}\right)\right]+\hat{\mu}_{2}(\omega)\left[\hat{\mu}_{3}(\omega)\left(k+L_{3}\right)\left(k\left(L_{2}-k-\left(k+L_{2}\right)\right)+\left(k^{2}+L_{2}^{2}\right) e^{2\left(k+L_{2}\right)}\right)\right. \\
& \left.\left.+k \hat{\mu}_{2}(\omega)\left(k^{2}-L_{2}^{2}\right)\left(1-e^{4 k}\right)\right]\right\} \\
& A_{32}^{(2)}=\frac{-i \omega e^{L_{2}}}{e^{2\left(L_{2}+k\right)} A_{0}}\left\{\hat{\mu}_{1}(\omega)\left(k-L_{1}\right) e^{2 k}\left[\hat{\mu}_{2}(\omega)\left(k^{2}-L_{2}^{2}\right)-\hat{\mu}_{3}(\omega)\left(k\left(k-L_{3}\right)+L_{2}\left(k-L_{3}\right)\right)\right]+e^{2 L_{2}}\right. \\
& \times\left[\left(L_{2}-k\right)\left(k \hat{\mu}_{2}(\omega)+L_{3} \hat{\mu}_{3}(\omega)\right)\left(\hat{\mu}_{1}(\omega)\left(k+L_{1}\right)+\hat{\mu}_{2}(\omega)\left(k+L_{2}\right)\right) e^{4 k}-k\left(\hat{\mu}_{2}(\omega)-\hat{\mu}_{3}(\omega)\right)\right. \\
& \left.\left.\times\left(k+L_{2}\right)\left(\hat{\mu}_{1}(\omega)\left(k+L_{1}\right)-\hat{\mu}_{2}(\omega)\left(k-L_{2}\right)\right)\right]\right\},
\end{aligned}
$$




$$
\begin{aligned}
& A_{41}^{(2)}=\frac{-i \omega e^{L_{2}}}{e^{2\left(L_{2}+k\right)} A_{0}}\left\{\hat{\mu}_{3}(\omega)\left(k+L_{3}\right) e^{2 k}\left[\hat{\mu}_{1}(\omega)\left(L_{1}\left(k-L_{2}\right)-k\left(k+L_{2}\right)\right)+\hat{\mu}_{2}(\omega)\left(k^{2}+L_{2}^{2}\right)\right]-k\left(k+L_{2}\right)\right. \\
& \times\left(\hat{\mu}_{1}(\omega)-\hat{\mu}_{2}(\omega)\right) e^{2 L_{2}}\left(\hat{\mu}_{2}(\omega)\left(k-L_{2}\right)-\hat{\mu}_{3}(\omega)\left(k+L_{3}\right)\right)-\left(k-L_{2}\right)\left(L_{1} \hat{\mu}_{1}(\omega)+k \hat{\mu}_{2}(\omega)\right) \\
& \left.\times\left(\hat{\mu}_{2}(\omega)\left(k+L_{2}\right)+\hat{\mu}_{3}(\omega)\left(k+L_{3}\right)\right) e^{2\left(2 k+L_{2}\right)}\right\}, \\
& A_{42}^{(2)}=\frac{i \omega e^{L_{2}}}{e^{2\left(L_{2}+k\right)} A_{0}}\left\{\hat{\mu}_{2}(\omega)\left(k^{2}-L_{2}^{2}\right)\left(k \hat{\mu}_{2}(\omega)\left(1-e^{4 k}\right)-\hat{\mu}_{3}(\omega)\left(k-L_{3} e^{4 k}\right)\right)-\hat{\mu}_{1}(\omega)\left(k+L_{1}\right)\left[\hat{\mu}_{2}(\omega)\right.\right. \\
& \times\left(k\left(k-L_{2}+\left(k+L_{2}\right) e^{4 k}\right)-\left(k^{2}+L_{2}^{2}\right) e^{2\left(k+L_{2}\right)}\right)+\hat{\mu}_{3}(\omega)\left(\left(k\left(k-L_{3}\right)-L_{2}\left(k+L_{3}\right)\right) e^{2\left(k+L_{2}\right)}\right. \\
& \left.\left.\left.+L_{3}\left(k+L_{2}\right) e^{4 k}-k\left(k-L_{2}\right)\right)\right]\right\} \text {, } \\
& A_{21}^{(3)}=\frac{i \omega \hat{\mu}_{2}(\omega)\left(k^{2}-L_{2}^{2}\right) e^{k}}{\left(k-L_{3}\right) e^{2\left(L_{2}+k\right)} A_{0}}\left\{\hat { \mu } _ { 1 } ( \omega ) \left[2 L_{2}\left(k+L_{1} e^{4 k}\right) e^{2 L_{2}}-\left(k\left(k+L_{2}\right)-L_{1}\left(k-L_{2}\right)\right) e^{2 k}+e^{2 k+4 L_{2}}\right.\right. \\
& \left.\left.\times\left(k\left(k-L_{2}\right)-L_{1}\left(k+L_{2}\right)\right)\right]-2 \hat{\mu}_{2}(\omega)\left[\left(k^{2}+L_{2}^{2}\right) \sinh \left(2 L_{2}\right)-2 k L_{2} \sinh (2 k)\right] e^{2\left(k+L_{2}\right)}\right\}, \\
& A_{22}^{(3)}=\frac{i \omega e^{k}}{2\left(k-L_{3}\right) e^{2\left(L_{2}+k\right)} A_{0}}\left\{4 \hat { \mu } _ { 2 } ( \omega ) ( k ^ { 2 } - L _ { 2 } ^ { 2 } ) e ^ { 2 ( k + L _ { 2 } ) } \left[\hat { \mu } _ { 2 } ( \omega ) \left(\operatorname { s i n h } ( 2 L _ { 2 } ) \left(k\left(k^{2}+L_{2}^{2}\right) \cosh (2 k)-L_{3}\right.\right.\right.\right. \\
& \left.\left.\times\left(k^{2}-L_{2}^{2}\right) \sinh (2 k)\right)-2 k^{2} L_{2} \cosh \left(2 L_{2}\right) \sinh (2 k)\right)-\hat{\mu}_{3}(\omega)\left(k^{2}+L_{3}^{2}\right)\left(k \cosh (2 k) \sinh \left(2 L_{2}\right)\right. \\
& \left.\left.-L_{2} \cosh \left(2 L_{2}\right) \sinh (2 k)\right)\right]+\hat{\mu}_{1}(\omega)\left(k+L_{1}\right)\left[\hat { \mu } _ { 2 } ( \omega ) \left(k^{4}\left(1-e^{4 k}\right)\left(1-e^{4 L_{2}}\right)+2 e^{2\left(k+L_{2}\right)}\left(2 k L_{2}\right.\right.\right. \\
& \times\left(3 k^{2}+L_{2}^{2}+3 k L_{2} \sinh (2 k) \sinh \left(2 L_{2}\right)-\left(3 k^{2}+L_{2}^{2}\right) \cosh (2 k) \cosh \left(2 L_{2}\right)\right)-2 L_{3}\left(k^{2}-L_{2}^{2}\right) \\
& \left.\left.\times\left(k \cosh (2 k) \sinh \left(2 L_{2}\right)-L_{2} \cosh \left(2 L_{2}\right) \sinh (2 k)\right)\right)\right)-\hat{\mu}_{3}(\omega)\left(k^{2}+L_{3}^{2}\right)\left(k^{2}\left(1-e^{4 k}\right)\left(1-e^{4 L_{2}}\right)\right. \\
& \left.\left.\left.+4 L_{2} e^{2\left(k+L_{2}\right)}\left(2 k\left(1-\cosh (2 k) \cosh \left(2 L_{2}\right)\right)+L_{2} \sinh (2 k) \sinh \left(2 L_{2}\right)\right)\right)\right]\right\} \text {, } \\
& A_{41}^{(3)}=\frac{-i \omega \hat{\mu}_{2}(\omega)\left(k^{2}-L_{2}^{2}\right) e^{L_{3}}}{\left(k-L_{3}\right) e^{2\left(L_{2}+k\right)} A_{0}}\left\{\hat { \mu } _ { 1 } ( \omega ) \left(2 L_{2}\left(k+L_{1} e^{4 k}\right) e^{2 L_{2}}+e^{2 k}\left(L_{1}\left(k-L_{2}\right)-k\left(k+L_{2}\right)\right)+e^{2\left(2 L_{2}+k\right)}\right.\right. \\
& \left.\left.\times\left(k\left(k-L_{2}\right)-L_{1}\left(k+L_{2}\right)\right)\right)-2 \hat{\mu}_{2}(\omega)\left(\left(k^{2}+L_{2}^{2}\right) \sinh \left(2 L_{2}\right)-2 k L_{2} \sinh (2 k)\right)\right\}, \\
& A_{42}^{(3)}=\frac{i \omega e^{L_{3}}}{\left(k-L_{3}\right) e^{2\left(L_{2}+k\right)} A_{0}}\left\{\hat { \mu } _ { 2 } ( \omega ) ( L _ { 2 } ^ { 2 } - k ^ { 2 } ) \left[\hat { \mu } _ { 2 } ( \omega ) \left(k L_{2}\left(1-e^{4 k}\right)\left(1+e^{4 L_{2}}\right)-k^{2}\left(1-e^{4 L_{2}}\right)-L_{2}^{2}\right.\right.\right. \\
& \left.\left.\times\left(1-e^{4 L_{2}}\right) e^{4 k}\right)+k \hat{\mu}_{3}(\omega)\left(k\left(1+e^{4 k}\right)\left(1-e^{4 L_{2}}\right)-L_{2}\left(1-e^{4 k}\right)\left(1+e^{4 L_{2}}\right)\right)\right]+\hat{\mu}_{1}(\omega)\left(k+L_{1}\right) \\
& \times\left[\hat { \mu } _ { 2 } ( \omega ) \left(L _ { 2 } \left(k\left(2 k-L_{2}+e^{4 L_{2}}\left(2 k+L_{2}\right)\right)+e^{4 k}\left(e^{4 L_{2}}\left(k-L_{2}\right)^{2}+\left(k+L_{2}\right)^{2}\right)-2\left(3 k^{2}+L_{2}^{2}\right)\right.\right.\right. \\
& \left.\left.\times e^{2\left(k+L_{2}\right)}\right)-k^{3}\left(1-e^{4 L_{2}}\right)\right)+k \hat{\mu}_{3}(\omega)\left[k^{2}\left(1-e^{4 k}\right)\left(1+e^{4 L_{2}}\right)+4 L_{2} e^{2\left(k+L_{2}\right)}(2 k(1-\cosh (2 k)\right. \\
& \left.\left.\left.\left.\times \cosh \left(2 L_{2}\right)+L_{2} \sinh (2 k) \sinh \left(2 L_{2}\right)\right)\right]\right]\right\} .
\end{aligned}
$$

Appendix B. The formulas for the quantities which are used in Equation (3.15) are

$$
\begin{aligned}
B_{11}^{(1)}= & \left(1 / B_{0}\right)\left\{2 ( 1 - \hat { \mu } _ { e 1 } ) H ^ { ( 1 ) } e ^ { 3 k } \left(\hat{\mu}_{e 2} \sin \left(\theta_{1}\right) \cosh (2 k)+\sin \left(\theta_{1}\right) \sinh (2 k)\right.\right. \\
& \left.\left.+i\left[\hat{\mu}_{e 2} \cos \left(\theta_{1}\right) \sinh (2 k)+\cos \left(\theta_{1}\right) \cosh (2 k)\right]\right)\right\}
\end{aligned}
$$




$$
\begin{aligned}
B_{0}= & \left(1+\hat{\mu}_{e 1}\right)\left(1+\hat{\mu}_{e 2}\right) e^{4 k}-\left(1-\hat{\mu}_{e 1}\right)\left(1-\hat{\mu}_{e 2}\right), \\
B_{12}^{(1)}= & \left(1 / B_{0}\right)\left\{2 H^{(1)} e^{3 k}\left(\hat{\mu}_{e 2}-1\right)\left(\hat{\mu}_{e 1} \sin \left(\theta_{1}\right)+i \cos \left(\theta_{1}\right)\right)\right\}, \\
B_{11}^{(2)}= & \left(1 / B_{0}\right)\left\{H^{(1)} e^{k}\left(\hat{\mu}_{e 1}-1\right)\left(1-\hat{\mu}_{e 2}\right)\left(\hat{\mu}_{e 1} \sin \left(\theta_{1}\right)-i \cos \left(\theta_{1}\right)\right)\right\}, \\
B_{12}^{(2)}= & \left(1 / B_{0}\right)\left\{H^{(1)} e^{3 k}\left(1+\hat{\mu}_{e 1}\right)\left(\hat{\mu}_{e 2}-1\right)\left(\hat{\mu}_{e 1} \sin \left(\theta_{1}\right)+i \cos \left(\theta_{1}\right)\right)\right\}, \\
B_{21}^{(2)}= & \left(1 / B_{0}\right)\left\{H^{(1)} e^{3 k}\left(1+\hat{\mu}_{e 2}\right)\left(\hat{\mu}_{e 1}-1\right)\left(\hat{\mu}_{e 1} \sin \left(\theta_{1}\right)-i \cos \left(\theta_{1}\right)\right)\right\}, \\
B_{22}^{(2)}= & \left(1 / B_{0}\right)\left\{H^{(1)} e^{k}\left(1-\hat{\mu}_{e 1}\right)\left(1-\hat{\mu}_{e 2}\right)\left(\hat{\mu}_{1} \sin \left(\theta_{1}\right)+i \cos \left(\theta_{1}\right)\right)\right\}, \\
B_{21}^{(3)}= & \left(1 / B_{0}\right)\left\{2 H^{(1)} e^{3 k}\left(\hat{\mu}_{e 1}-1\right)\left(\hat{\mu}_{e 1} \sin \left(\theta_{1}\right)-i \cos \left(\theta_{1}\right)\right)\right\}, \\
B_{22}^{(3)}= & \left(\left(1-\hat{\mu}_{e 2}\right) H^{(1)} e^{k} / \hat{\mu}_{e 2} B_{0}\right)\left\{\left(1+\hat{\mu}_{e 1}\right)\left(\hat{\mu}_{e 1} \sin \left(\theta_{1}\right)-i \hat{\mu}_{e 2} \cos \left(\theta_{1}\right)\right) e^{4 k}\right. \\
& \left.+\left(\hat{\mu}_{e 1}-1\right)\left(\hat{\mu}_{e 1} \sin \left(\theta_{1}\right)+i \hat{\mu}_{e 2} \cos \left(\theta_{1}\right)\right)\right\} .
\end{aligned}
$$

Appendix C. The values of $v$ 's which are used in relation (4.5) are

$$
\begin{aligned}
& v_{1}=\alpha_{11}, \\
& v_{2}=\alpha_{11}\left(\alpha_{11} \alpha_{21}+\alpha_{12} \alpha_{22}-\alpha_{31}\right)-\alpha_{22}^{2}, \\
& v_{3}=v_{2}\left(\alpha_{11}\left(\alpha_{21} \alpha_{31}+\alpha_{22} \alpha_{32}-\alpha_{11} \alpha_{41}-\alpha_{12} \alpha_{42}\right)-\alpha_{31}^{2}\right) \\
& -\left(\alpha_{11}\left(\alpha_{11} \alpha_{32}-\alpha_{12} \alpha_{31}\right)-\alpha_{11} \alpha_{42}+\alpha_{22} \alpha_{31}\right)^{2} \text {, } \\
& v_{4}=v_{41} v_{42}-v_{43}^{2},
\end{aligned}
$$

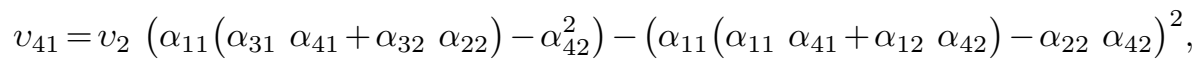

$$
\begin{aligned}
& v_{42}=v_{2}\left(\alpha_{11}\left(\begin{array}{lll}
\alpha_{31} & \alpha_{21}+\alpha_{32} & \alpha_{22}
\end{array}\right)-\alpha_{11}\left(\alpha_{11} \alpha_{41}+\alpha_{12} \alpha_{41}\right)-\alpha_{31}^{2}\right) \\
& -\left(\alpha_{11}\left(\alpha_{11} \alpha_{32}-\alpha_{12} \alpha_{31}\right)-\alpha_{11} \alpha_{42}+\alpha_{22} \alpha_{31}\right)^{2} \text {, }
\end{aligned}
$$

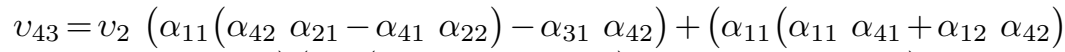

$$
\begin{aligned}
& \left.-\alpha_{42} \alpha_{22}\right)\left(\alpha_{11}\left(\alpha_{11} \alpha_{32}-\alpha_{12} \alpha_{31}\right)-\alpha_{11} \alpha_{42}+\alpha_{31} \alpha_{22}\right) \text {. }
\end{aligned}
$$




\section{REFERENCES}

[1] T. Funada and D.D. Joseph, Viscous potential flow analysis of Kelvin-Helmholtz instability in a channel, J. Fluid Mech., 445, 263-283, 2001.

[2] P.K. Bhatia, Rayleigh-Taylor instability of two viscous superposed conducting fluids, Nuovo Cimento, 19B, 161-168, 1974.

[3] P.G. Drazin and W.H. Reid, Hydrodynamic Stability, Univ. Press, Cambridge, 1981.

[4] D.D. Joseph, Stability of Fluid Motions II, Springer-Verlag, New York, 1976.

[5] A.F. Elhefnawy, Intervals of an unsteady electrohydrodynamic Kelvin-Helmoltz stability, Physica A, 214, 229-241, 1995.

[6] K. Zakaria, M.A. Sirwah, and S. Alkharashi, Temporal stability of superposed magnetic fluids in porous media, Physica Scripta, 77, 1-20, 2008

[7] K. Zakaria, M.A. Sirwah, and S. Alkharashi, Instability through porous media of three layers superposed conducting fluids, European J. Mech. B/Fluids, 28, 259-270, 2009.

[8] R.G. Larson, Constitutive Equations for Polymer Melts and Solutions, Butterworths, Boston, 1988.

[9] Z. Liu, G. Brenn, and F. Durst, Linear analysis of the instability of two-dimensional nonNewtonian liquid sheets, J. Non-Newtonian Fluid Mech., 78, 133-166, 1998.

[10] M. Akcay and M.A. Yukselen, Drag reduction of a non-Newtonian fluid by fluid injection on a moving wall, Arch. Appl. Mech., 69, 215-225, 1999.

[11] A.M. Siddiqui, T. Hayat, and S. Asghar, Periodic flows of a non-Newtonian fluid between two parallel plates, Internat. J. Non-Linear Mech., 34, 895-899, 1999.

[12] G. Brenn, Z. Liu, and F. Durst, Linear analysis of the temporal instability of axisymmetrical non-Newtonian liquid jets, Int. J. Multiphase Flow, 26, 1621-1644, 2000.

[13] W.C. Tan and T. Masuoka, Stokes first problem for an Oldroyd-B fluid in a porous half space, Phys. fluid, 17, 023101-023107, 2005.

[14] H. Hadim, Non-Darcy natural convection of a non-Newtonian fluid in a porous cavity, Int. Comm. in Heat and Mass Transfer, 33, 1179-1189, 2006.

[15] M. Khan, M. Saleem, C. Fetecau, and T. Hayat, Transient oscillatory and constantly accelerated non-Newtonian flow in a porous medium, Int. J. of Non-Linear Mech., 42, 1224-1239, 2007.

[16] Z. Liu, Instability of a viscoelastic liquid jet with axisymmetric and asymmetric disturbances, Int. J. of Multiphase Flow, 34, 42-60, 2008.

[17] L. Sheu, L. Tam, J. Chen, H. Chen, K. Lin, and Y. Kang, Chaotic convection of viscoelastic fluids in porous media, Chaos, Solitons and Fractals, 37, 113-124, 2008.

[18] T. Hayat, M. Khan, M. Ayub, and M. Siddiqui, The unsteady Couette flow of a second grade fluid in a layer of porous medium, Arch. Mech., 57, 405-416, 2005.

[19] P. Kumar and G.J. Singh, Stability of two superposed Rivlin-Ericksen viscoelastic fluids in the presence of suspended particles, Rom. J. Phys., 51, 927-935, 2006.

[20] W. Tan and T. Masuoka, Stability analysis of a Maxwell fluid in a porous medium heated from below, Physics Letters A, 360, 454-460, 2007.

[21] B. Khuzhayorov, J.L. Auriault, and P. Royer, Derivation of macroscopic filtration law for transient linear viscoelastic fluid flow in porous media, Int. J. Eng. Sci., 38, 487-504, 2000 .

[22] M. Saghir, P. Comi, and M. Mehrvar, Effects of interaction between Rayleigh and Marangoni convection in superposed fluid and porous layers, Int. J. Therm. Sci., 41, 207-215, 2002.

[23] D. Nield, The stability of flow in a channel or duct occupied by a porous medium, Int. J. of Heat and Mass Transfer, 46, 4351-4354, 2003.

[24] R.E. Rosensweig, Ferrohydrodynamics, Cambridge, Cambridge University Press, 1985.

[25] H.H. Woodson and J.R. Melcher, Electromechanical Dynamics, John Wiley, Sons., 1968.

[26] J.R.A. Pearson and P.M.J. Tardy, Models for flow of non-Newtonian and fluids through porous media, J. Non- Newtonian Fluid Mech., 102, 447-473, 2002.

[27] L. Wu and S.Y. Chou, Electrohydrodynamic instability of a thin film of viscoelastic polymer underneath a lithographically manufactured mask, J. Non-Newtonian Fluid Mech., 125, 91-99, 2005.

[28] Sunil, R.C. Sharma, and R.S. Chandel, On superposed couple-stress fluids in porous medium in hydromagnetics, Z. Naturforsch, 57a, 955-960, 2002.

[29] M. Goldin, J. Yerushalmi, R. Pfeffer, and R. Shinnar, Breakup of a laminar capillary jet of a viscoelastic fluid, J. Fluid Mech., 38, 689-711, 1969.

[30] O. Ozen, N. Aubry, D.T. Papageorgiou, and P.G. Petropoulos, Electrohydrodynamic linear stability of two immiscible fluids in channel flow, Electrochimica Acta, 51, 5316-5323, 2006 . 